\title{
An integrated approach for airline scheduling, aircraft fleeting and routing with cruise speed control
}

\author{
Hüseyin Gürkan ${ }^{\mathrm{a}}$, Sinan Gürel ${ }^{\mathrm{b}}$, M. Selim Aktürk ${ }^{\mathrm{c}, *}$ \\ ${ }^{a}$ Fuqua School of Business, Decision Sciences, Duke University, USA \\ ${ }^{\mathrm{b}}$ Department of Industrial Engineering, Middle East Technical University, Turkey \\ ${ }^{\mathrm{c}}$ Department of Industrial Engineering, Bilkent University, Turkey
}

\section{A R T I C L E I N F O}

\section{Article history:}

Received 12 October 2015

Received in revised form 5 March 2016

Accepted 5 March 2016

Available online 1 April 2016

\section{Keywords:}

Airline scheduling

Aircraft fleeting and routing

Cruise time controllability

Second order cone programming

\begin{abstract}
A B S T R A C T
To place an emphasis on profound relations among airline schedule planning problems and to mitigate the effect of unexpected delays, we integrate schedule design, fleet assignment and aircraft routing problems within a daily planning horizon while passengers' connection service levels are ensured via chance constraints. We propose a nonlinear mixed integer programming model due to the nonlinear fuel consumption and $\mathrm{CO}_{2}$ emission cost terms in the objective function, which is handled by second order conic reformulation. The key contribution of this study is to take into account the cruise time control for the first time in an integrated model of these three stages of airline operations. Changing cruise times of flights in an integrated model enables to construct a schedule to increase utilization of fuel efficient aircraft and even to decrease total number of aircraft needed while satisfying the same service level and maintenance requirements for aircraft fleeting and routing. There is a critical tradeoff between the number of aircraft needed to fulfill the required flights and overall operational expenses. We also propose two heuristic methods to solve larger size problems. Finally, computational results using real data obtained from a major U.S. carrier are presented to demonstrate potential profitability in applying the proposed solution methods.
\end{abstract}

(c) 2016 Elsevier Ltd. All rights reserved.

\section{Introduction}

Managing an airline is unavoidably expensive. One of the most basic costs is the price of purchasing the aircraft which range from 70 to 400 mio USD per unit Boeing (2015). Moreover, among the operational expenses, fuel has been the largest single cost term for the global airlines. According to IATA (2010) analysis on airline financial data, fuel expenses accounted for $\$ 210$ billion in 2013 for global airline industry. On the other hand, unexpected delays are endemic in airline operations and to demonstrate their impact, the estimated total cost to the U.S. economy because of flight delays was as much as $\$ 41$ billion in 2007 Rebollo and Balakrishnan (2014). Naturally, the efficient utilization of such expensive resources, decreasing operational expenses and higher robustness are objectives of any profitable airline. However, unfortunately these terms are inversely correlated, in other words higher utilization might cause higher operational cost, less robustness and vice versa. In this direction, generating a robust schedule with high aircraft utilization and less operational expenses at the same time is quite crucial for any airline. Therefore, in this paper, we propose an integrated model for robust schedule design, aircraft

\footnotetext{
* Corresponding author.
} 
routing and fleeting with cruise speed control that aims efficient aircraft utilization and robustness within the consideration of operational expenses such as fuel consumption, $\mathrm{CO}_{2}$ emission and spill cost.

Airline schedule design problem decides where to fly and in which frequency in consideration of market demand, profitability, available resources and the competitors. Due to its broad scope, Barnhart et al. (2003) state that building flight schedules from scratch is performed manually with limited optimization in the typical airline practice. Following the construction or design of a flight schedule, fleet assignment problem tries to find the optimal assignment of aircraft types to flights by considering the number of aircraft in each fleet and coverage of all flights. After fleet assignment decomposes flight networks into subnetworks in terms of a particular fleet type, maintenance routing problem assigns individual aircraft to these flights in consideration of the maintenance requirements. For a general review on airline schedule planning problems, we refer the reader to Barnhart and Cohn (2004).

Since the different airline schedule planning problems are strongly related to each other, several integrated models are proposed that take into account combinations of these problems to improve suboptimal solutions for the entire system. Lohatepanont and Barnhart (2004) consider schedule design and fleet assignment in an integrated way in which a base schedule and two flight lists including mandatory and optional flights are given. Starting from the base schedule they consider deleting/adding flights from/to the base schedule with respect to given flight lists. In a similar fashion, Sherali et al. (2013a) propose a model that integrates the schedule design and fleet assignment processes while considering flexible flight times, schedule balance, and recapture issues, along with optional legs, path/itinerary-based demands, and multiple fareclasses. Differently, they consider the flow of passengers along itineraries over the network together with flight scheduling and fleeting decisions in order to maximize profits. Integrating three problems enables to improve local optimal solutions, however tractability worsens as much as the scope of integration expands. Therefore, these integrated problems are modeled and solved for a daily planning horizon.

In the literature, there are studies related to our work in some aspects such as daily planning horizon, passenger connection, cruise speed control or maintenance considerations. Duran et al. (2015) propose a robust airline scheduling model with controllable cruise times. In their study, the tradeoff between the costs of cruise time change and idle time insertion is considered while passengers' connection service levels are ensured by chance constraints. Speed control is quite a recent concept in solving airline scheduling problems. Aktürk et al. (2014) is the first study that makes use of speed control in the context of airline schedule recovery from disruptions. Sherali et al. (2013b) propose an approach in which they integrate the schedule design, fleet assignment, and aircraft-routing problems within the consideration of flight selection, departure timing and maintenance requirements. For maintenance requirements, they use a limit on total flight time of each aircraft that might be different for each fleet type. As a solution method, they use Benders' decomposition and enhance the model via valid inequalities. Haouari et al. (2013) propose a model for daily maintenance routing problem in which they ensure maintenance feasibility by counter constraints on flight hours, take offs and number of days since the last maintenance checks for each aircraft. They present a compact polynomial-sized representation for the general aircraft routing model and they linearize and lift that representation. Moreover, in the study of Aloulou et al. (2013), a MIP model is proposed for the robust aircraft routing problem without directly accommodating maintenance constraints however by considering that the flights start and end in the single hub where maintenance checks are achieved overnight. Aloulou et al. (2013) capture robustness by an objective function pertaining to aircraft and passenger connections.

What distinguishes our work from the studies above and makes challenging simultaneously is cruise speed control and integration. To the best of our knowledge, this is the first study in which cruise speed/time is controlled within the integrated robust schedule design, aircraft fleeting and routing problem. In our study, the fuel consumption and $\mathrm{CO}_{2}$ emission cost functions are nonlinear functions in cruise time and involve binary variables. We have shown that these nonlinear functions with binary variables can be transformed into a set of second order conic inequalities. Moreover, even if it is a special case of our problem, Parmentier (2013) showed that aircraft routing problem by itself is an NP-complete problem. In addition to aircraft routing problem, we consider robust airline scheduling and fleet type assignment problems in an integrated fashion that involves a large number of decision variables. For that reason, when the number of flights and aircraft increases, the problem size increases drastically. We also consider passengers' connection service levels with chance constraints as well as departure timing, idle time insertion and cruise speed control different from the aircraft routing problem. Changing cruise time of flights in an integrated model enlarges the solution space and enables to construct a schedule with new flight sequences, which could not be considered previously due to fixed cruise speed/time restriction. For two flights to be connected consecutively by the same aircraft, there must be enough time gap between departure times of these flights. This time gap is the sum of cruise time, non-cruise time, turnaround time and idle time. In other studies, the lower bound for this gap is taken as fixed, however cruise speed/time change enables to control this lower bound on the gap between departure times. By this means, in our study more flight connection alternatives could be generated.

The first contribution brought by our study is that aircraft utilization could be increased and even total number of aircraft needed to cover a set of flights could be decreased while ensuring equivalent service level and maintenance requirements. Due to having more alternatives on flight connections and compression of cruise time of flights, it is possible to increase the number of flights to be performed by an efficient aircraft. While this increase in the utilization of fuel efficient aircraft could reduce the minimum number of required aircraft to perform a set of flights. There is a critical tradeoff between the number of aircraft needed to fulfill the required flights and the overall operational expenses, such as fuel consumption costs.

The second is the robustness issue. Since we have more alternatives on flight connections, it is possible to generate better flight sequences in terms of robustness. For example, on a route having a flight with a great delay probability would require 
an intervention for the following flights to be performed on time while removing the problematic flight from that sequence could render that intervention unnecessary. Our study has more options to make this type of changes on routing decisions so changing routes could improve the robustness.

Finally, we have used the recent advances in second order cone programming to handle the nonlinear mixed integer programming formulation. Furthermore, in order to solve the large scale problems in a reasonable time, we propose two heuristic algorithms. The first one is discretized approximation and cruise speed control algorithm, and the second one is multi-stage triplet search algorithm.

In the remaining of the paper, in Section 2, the problem definition is presented. Section 3 is devoted to the problem formulation and conic reformulation. In Section 4, heuristic methods are proposed and in Section 5 results of a computational study are presented.

\section{Problem definition}

Our problem is to solve robust airline schedule design, aircraft fleeting and routing problems within a daily planning horizon for a given set of flights and a set of aircraft in an integrated manner while considering maintenance requirements and passengers' connection service levels. In the proposed model, we determine the departure time, cruise time, an inserted idle time, if necessary, and aircraft fleet type for each flight along with the routing decisions. For each aircraft, the model determines whether that aircraft is going to be used and, if it is used, the flight sequence to be flown for that particular aircraft. These decisions are made in consideration of the idle time insertion cost, fuel consumption and $\mathrm{CO}_{2}$ emission costs, spill cost and aircraft usage cost simultaneously.

The feasible set of solutions satisfy maintenance requirements, passengers' connection service levels along with the flight connection constraints in cases where two flights are performed consecutively by the same aircraft. For maintenance requirements, we adopt two basic rules for each aircraft. The first one is that we limit the total cruise time for each aircraft on a day. A similar approach is used by Sherali et al. (2013b), they use a limit $\left(\lambda_{t}\right)$ on total flight hours of each aircraft on a day. The second one is that the first and the last airport of an aircraft is predetermined on a day similar to the study of Aloulou et al. (2013). In case when an aircraft is used, in order that aircraft to follow its ordinary maintenance checks, we secure that aircraft takes off from/lands to the particular airports at the beginning/end of a day. For passengers' connection, we use service levels with chance constraints and ensure necessary time for passengers to make connection with a particular probability, denoted as service level, between each pair of flights which is feasible for passengers' connection. Furthermore, we provide minimum turnaround time between flights which are performed by the same aircraft.

Similar to the study of Duran et al. (2015), in our model we take the flight time as the sum of cruise time and non-cruise time. Non-cruise time consists of the taxi-in and taxi-out stages as well as climb and descend stages of a flight, which include uncertainty depending on the airport congestion or weather conditions while cruise time refers to the time which is controllable by with speeding up the aircraft. Consequently, we take cruise time as a decision variable and non-cruise time as a given parameter. On the other hand, there are different methods in the literature to investigate the role of fuel cost on aircraft operating costs as discussed by Ryerson and Hansen (2013). In this study, we have used the following function proposed by Aktürk et al. (2014) and EUROCONTROL (2012) to calculate fuel consumption in $\mathrm{kg}$ for a given cruise time of flight $i$ operated by aircraft type $t$ :

$$
F_{i}^{t}\left(f_{i}^{t}\right)=c_{1}^{i, t} \cdot \frac{1}{f_{i}^{t}}+c_{2}^{i, t} \cdot \frac{1}{\left(f_{i}^{t}\right)^{2}}+c_{3}^{i, t} \cdot\left(f_{i}^{t}\right)^{3}+c_{4}^{i, t} \cdot\left(f_{i}^{t}\right)^{2}
$$

where $c_{1}^{i, t}, c_{2}^{i, t}, c_{3}^{i, t}$ and $c_{4}^{i, t}$ correspond to aircraft properties and the coefficients related to physical conditions of a flight for each flight $i$ and aircraft type $t$ pair as discussed in Aktürk et al. (2014). These coefficients for 399 different aircraft types are available in EUROCONTROL (2012). Fuel cost has been the largest operational expense for the global airlines. The EU emissions trading system (EU ETS) covers airlines as well as power plants and industrial plants in 31 countries. By introducing $\mathrm{CO}_{2}$ emission costs, these trading systems aim at cutting $\mathrm{CO}_{2}$ emissions by airline companies. Consequently, the fuel consumption and $\mathrm{CO}_{2}$ emission costs are calculated by multiplying fuel consumption with their corresponding cost coefficients $\left(c_{\text {fuel }}+c_{\mathrm{CO}_{2}}\right)$.

In this study, similar to the study of Duran et al. (2015), passengers' connections are taken into account to develop a robust schedule such that mis-connections of passengers are minimized when a disruption occurs. Between two flights $i$ and $j$, if the origin airport of the flight $j$ is same as the destination airport of the flight $i$ and the departure time of flight $j$ is later than the arrival time of the flight $i$, the time needed for the passengers' connection is $T P_{i j}$. It is ensured that the percentage of the passengers' connection satisfied between flights $(i, j)$ is greater than the minimum desired service level for each connecting flight pair, denoted as $\gamma_{i j}$, through a set of probabilistic chance constraints as discussed later.

\subsection{Numerical example}

In order to elaborate our problem definition and the model mechanics, in this section, we provide a numerical example. First, we present a published schedule in Table 1, which shows the planned departure time and cruise time for each flight, and idle time after each flight, if necessary, to satisfy the passenger connections. In our proposed approach, a new schedule 
Table 1

Published schedule.

\begin{tabular}{|c|c|c|c|c|c|c|}
\hline Tail\# & Flight \# & From & To & Departure time & Cruise time & Idle time \\
\hline \multirow[t]{2}{*}{ N3ELAA } & 2057 & ORD & SJU & $08: 30$ & 270 & 35.6 \\
\hline & 2078 & SJU & ORD & $14: 25$ & 315 & - \\
\hline \multirow[t]{3}{*}{ N3DUAA } & 2099 & ORD & LAX & 07:00 & 250 & 34.9 \\
\hline & 1972 & LAX & ORD & $12: 40$ & 225 & 24.4 \\
\hline & 1972 & ORD & RDU & $17: 45$ & 95 & - \\
\hline \multirow[t]{2}{*}{ N412AA } & 2345 & ORD & DFW & $17: 15$ & 135 & 2.5 \\
\hline & 2374 & DFW & ORD & $20: 40$ & 110 & - \\
\hline \multirow[t]{3}{*}{ N4XGAA } & 2079 & ORD & SAN & $08: 45$ & 250 & 13.5 \\
\hline & 1438 & SAN & ORD & $14: 00$ & 230 & 58.9 \\
\hline & 346 & ORD & LGA & $19: 50$ & 115 & - \\
\hline
\end{tabular}

with a new aircraft fleeting and routing is generated by considering idle time cost, fuel consumption and $\mathrm{CO}_{2}$ emission costs, spill cost and daily usage cost simultaneously. In accordance with the marketing requirements, the departure times could be changed within $\pm 15 \mathrm{~min}$. The proposed schedule, given in Table 2 , is feasible in terms of maintenance requirements because all aircraft land to the same airports as in the published schedule, and we also limit the flight hours of each aircraft regarding maintenance requirements. Further, passengers' service levels are ensured by the chance constraints in the proposed schedule. For this numerical example same service level is adopted with the published schedule for each passengers' connection.

There are major changes between two schedules such as the number of aircrafts used, routings of aircraft, idle time insertion and cruise times. The total number of aircraft used in the published schedule was four, while the proposed schedule clearly illustrates that the same passenger connection service levels can be attained with three aircraft. This improvement can be achieved by compressing the cruise time of flight 1438 around 14\%, from $230 \mathrm{~min}$ to $198.4 \mathrm{~min}$ in the proposed schedule. In addition, the departure time of flight 2057 is changed from 8:30 to 8:45 in the proposed schedule. Fig. 1 represents the time space networks of the schedules. The horizontal axis represents the time and the vertical axis represents the airports. For each flight, there is an angular arrow nearby and a flight number. The horizontal arrows represent the turn around and idle time of an aircraft in the corresponding airport. The route of each aircraft is drawn by a different line style. For example, the solid arrows represent the operations of tail number N4XGAA.

We compare the performance of the published schedule with the proposed schedule in terms of the improvement in total cost by using the following formula:

$$
\text { Cost Improvement }=100 \times \frac{\text { Cost of the Published Schedule }- \text { Cost of the Proposed Schedule }}{\text { Cost of the Published Schedule }}
$$

The cost calculation of the published schedule is shown in Table 3 . The fuel consumption and $\mathrm{CO}_{2}$ emission costs calculations are explained in former sections. Idle time costs are calculated by the multiplication of unit idle time cost of each aircraft and the idle time amount of that aircraft after each flight. For a given demand realization, spill costs are calculated by multiplication of the number of spilled passengers and the cost incurred when a spill occurs. In the published schedule, we assume that there is no spill cost. However, when we assign an aircraft with a smaller number of seats than the number of already assigned passengers, a spill cost can incur as shown in Table 4. Daily usage costs are the costs incurred when the corresponding aircraft are used. Total cost of the published schedule is $\$ 419,821.3$.

As mentioned before, the total number of aircraft used is decreased from 4 to 3 . In the proposed model, the most expensive aircraft in terms of daily usage cost is N3ELAA which is not a fuel efficient aircraft in comparison to N412AA and N3DUAA. Fig. 2 illustrates the differences in efficiency by providing the fuel consumption and $\mathrm{CO}_{2}$ emission costs that would occur if flight 1438 was performed by these aircraft. Our model generates a schedule in which N3ELAA is not used due to its high daily usage cost and low fuel efficiency while the other aircraft are utilized more. Although the fuel efficiency of aircraft

Table 2

Proposed schedule.

\begin{tabular}{|c|c|c|c|c|c|c|}
\hline Tail\# & Flight \# & From & To & Departure time & Cruise time & Idle time \\
\hline \multirow[t]{3}{*}{ N3DUAA } & 2079 & ORD & SAN & 08:38 & 249.5 & 8.4 \\
\hline & 1438 & SAN & ORD & $13: 45$ & 198.4 & - \\
\hline & 1972 & ORD & RDU & $18: 00$ & 94.8 & - \\
\hline \multirow[t]{4}{*}{ N412AA } & 2099 & ORD & LAX & $06: 55$ & 249.9 & - \\
\hline & 1972 & LAX & ORD & $12: 25$ & 221.7 & - \\
\hline & 2345 & ORD & DFW & $17: 26$ & 133 & - \\
\hline & 2374 & DFW & ORD & $20: 55$ & 110 & - \\
\hline \multirow[t]{3}{*}{ N4XGAA } & 2057 & ORD & SJU & $08: 45$ & 275.9 & 1.2 \\
\hline & 2078 & SJU & ORD & $14: 10$ & 293.6 & - \\
\hline & 346 & ORD & LGA & $20: 05$ & 115 & - \\
\hline
\end{tabular}




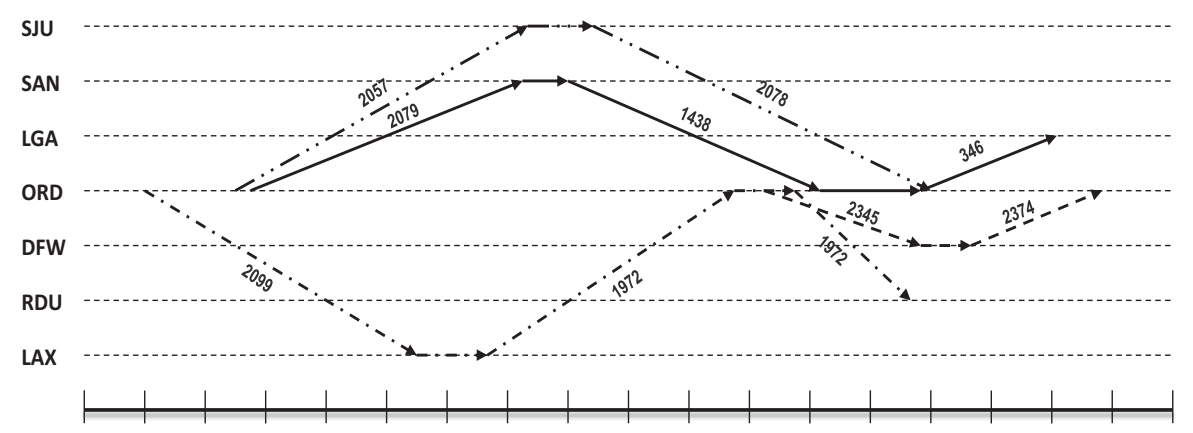

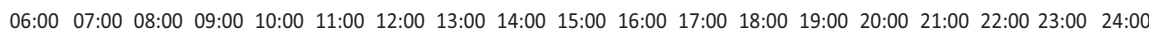

(a) Published schedule

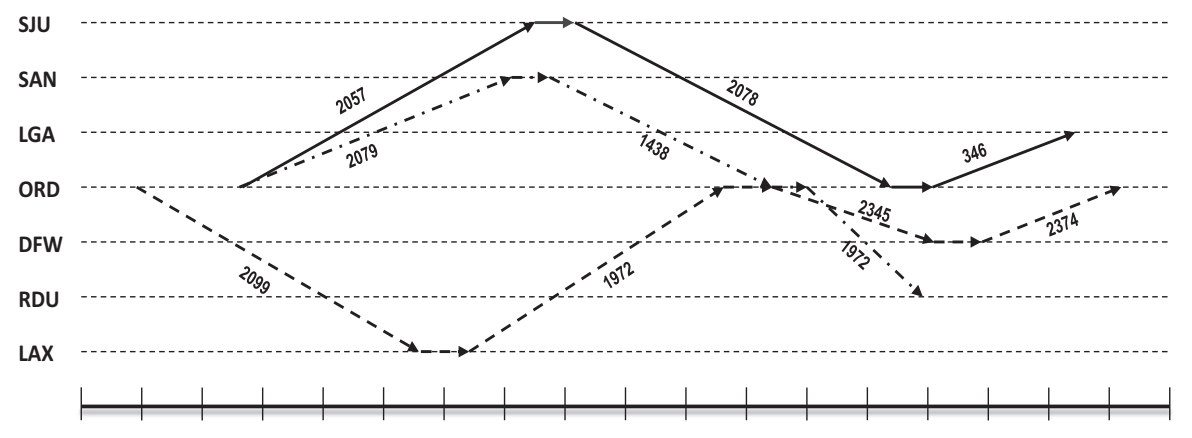

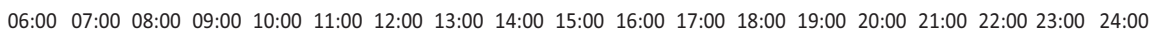

(b) Proposed schedule

Fig. 1. Time space networks.

Table 3

Cost calculation for the published schedule.

\begin{tabular}{|c|c|c|c|c|c|}
\hline Tail\# & Flight \# & Fuel and $\mathrm{CO}_{2}$ & Idle time & Spill & Daily usage \\
\hline N3ELAA & $\begin{array}{l}2057 \\
2078\end{array}$ & $\begin{array}{l}7611 \\
8879.5\end{array}$ & $\begin{array}{l}5334 \\
-\end{array}$ & - & 90,000 \\
\hline N3DUAA & $\begin{array}{l}2099 \\
1972 \\
1972\end{array}$ & $\begin{array}{l}5923.4 \\
5331 \\
2250.9\end{array}$ & $\begin{array}{l}4955.8 \\
3461.9 \\
-\end{array}$ & $\begin{array}{l}- \\
- \\
-\end{array}$ & 85,200 \\
\hline N412AA & $\begin{array}{l}2345 \\
2374\end{array}$ & $\begin{array}{l}2319 \\
1889.6\end{array}$ & $\begin{array}{l}347.2 \\
-\end{array}$ & - & 84,000 \\
\hline N4XGAA & $\begin{array}{r}2079 \\
1438 \\
346\end{array}$ & $\begin{array}{l}6509.4 \\
5988.7 \\
2994.3\end{array}$ & $\begin{array}{l}1944 \\
8481.6 \\
-\end{array}$ & $\begin{array}{l}- \\
- \\
-\end{array}$ & 86,400 \\
\hline Total & & 49696.8 & 24524.5 & 0 & 345,600 \\
\hline
\end{tabular}

N4XGAA is worse than N3ELAA, the model prefers to use N4XGAA since its daily usage cost is smaller than N3ELAA. As a direct consequence of better aircraft utilization, the cost of daily usage is improved by $26 \%$.

Another effect of higher efficient aircraft utilization is on the fuel consumption and $\mathrm{CO}_{2}$ emission costs. Since utilizations of efficient aircraft are increased and better flight aircraft assignment is achieved, total cost of fuel consumption and $\mathrm{CO}_{2}$ emission is improved around 10\%. This improvement is achieved even if the cruise times of flights 2078,1438 and 2345 are decreased by $7 \%, 14 \%$ and $2 \%$, respectively. As an example of better flight aircraft assignment, in the published schedule flight 1438 is performed by aircraft N4XGAA with $\$ 5988.7$ fuel and emission cost, while in the proposed schedule same flight is performed by aircraft N3DUAA with $\$ 5650$ fuel and emission costs though the cruise time is compressed by $14 \%$ in albeit of additional fuel and emission costs. Since N3DUAA is more efficient than N4XGAA, the extra fuel and emission costs caused by that compression which is necessary to assign 1438 to N3DUAA can be compensated. In the proposed schedule, we increase the utilization of N412AA, which is the most fuel efficient aircraft. Furthermore, the necessity of idle time insertion after flights to satisfy the desired minimum service levels for passenger connections can be eliminated by changing routing decisions, cruise time and departure time. 
While there is an improvement in sum of daily usage cost, idle time cost, fuel consumption and $\mathrm{CO}_{2}$ emission costs, the spill cost is increased in the proposed schedule. The reason of this increase is that the fleet assignment in the published schedule is generated in consideration of aircraft capacity and passenger demand, however, in the proposed model the other cost terms are also considered. The improvement in the other cost terms overcomes the increase in the spill cost. While in the published schedule the spill cost is 0 , the proposed model has a spill cost $\$ 2022.1$. The spill of passengers are occurred in flights 2079, 1438, 2099 and 1972 in the proposed schedule.

When we compare the overall results, the total cost is improved $27 \%$ in the proposed schedule. The costs of both schedules are calculated at the planning stage. After the realizations of non-cruise times of each flight, the actual cost of the idle time and delay costs can be seen. For this purpose a simulation study can be done to compare the realized costs of the schedules. However, with this numerical example, we aim to show how the mechanics of the proposed model work.

\title{
3. Problem formulation
}

Our main aim in this paper is to integrate schedule design, fleeting and routing decisions along with aircraft cruise speed decisions so that the total cost is minimized while the passenger connection service level constraints are satisfied. In this section, we first present the mathematical formulation of the problem. Consequently, by the help of this model, we can easily demonstrate the interdependencies among these interrelated problems. Afterwards, we show the conic reformulation of the objective function and give the reformulation of the model.

\author{
Parameters \\ Idle $e_{t}$ Unit cost of the idle time of aircraft $t$ \\ $\mathrm{Cap}_{t}$ : Seat capacity of aircraft $t$ \\ $\operatorname{Dem}_{i}$ : Passenger demand of flight $i$ \\ Daily $_{t}$ : The cost incurred when aircraft $t$ is used \\ $T A_{i j}^{t}$ : Turntime needed to prepare aircraft $t$ between flights $i$ and $j$ \\ $T P_{i j}$ : Turnaround time needed to connect passengers between flights $i$ and $j$ \\ $\operatorname{Cspl}_{i}$ : Opportunity cost of spilled passengers of flight $i$ \\ $\lambda_{t}$ : The total available cruise time of aircraft $t$ on a day \\ $\gamma_{i j}$ : minimum desired passengers' connection service level between flights $i$ and $j$ \\ $c_{\text {fuel }}$ : Cost of fuel per $\mathrm{kg}$ of aircraft fuel consumption \\ $c_{\mathrm{CO}_{2}}$ : Cost of emission per $\mathrm{kg}$ of aircraft $\mathrm{CO}_{2}$ emission \\ $f_{i}^{u}, f_{i}^{l}$ : Upper and lower limit of the cruise time of flight $i$ \\ $d_{i}^{u}, d_{i}^{l}$ : Upper and lower limit of the departure time of flight $i$ \\ $e_{b}$ : Airport congestion coefficient for airport $b$ \\ $\mathrm{Or}_{i}$ : Origin airport of flight $i$ \\ $D n_{i}$ : Destination airport of flight $i$ \\ $N C_{i}$ : The random parameter denoting the non cruise time of flight $i$ \\ Decision variables \\ $x_{i j}^{t}: 1$ if flights $i$ and $j$ are performed by the same aircraft $t$ consecutively and is 0 ow. \\ $y_{i}^{t}: 1$ if flight $i$ is the first flight performed by aircraft $t$ and is 0 ow. \\ $z_{i}^{t}: 1$ if flight $i$ is the last flight performed by aircraft $t$ and is 0 ow. \\ $d_{i}$ : Departure time of flight $i$ \\ $s_{i}^{t}$ : Idle time of aircraft $t$ after flight $i$ \\ $f_{i}^{t}$ : Cruise time of flight $i$ performed by aircraft $t$
}

In the proposed nonlinear mixed integer programming model, we utilize the following sets. $T$ represents the set of aircraft that can be operated. $F$ is the set of flight legs which have to be covered by using the aircraft in $T$. For each flight $i$ in $F$, there are two sets which are $U^{i}$, upstream flights and $D^{i}$, downstream flights. $U^{i}$ denotes the set of flights which can follow flight $i$ in terms of departure time and origin, destination airports pair. It is easy to check origin destination pairs, since the destination airport of a flight in $U^{i}$ has to be same with the origin airport of $i$. For departure time, there has to be a sufficient time for connection so that a flight in $U^{i}$ and flight $i$ might be performed by the same aircraft consecutively. If flight $j$ is in $U^{i}$ then, flight $i$ is in $D^{j}$. A denotes the set of flights $(i, j)$ such that flight $j \in D^{i}$, i.e. flight $i \in U^{j}$. If $(i, j)$ is in $A$, it means that an aircraft can fly these flights consecutively. On the other hand, the connection $(i, j)$ can also be avoided removing this pair from the set $A$, whenever there are some conditions on $(i, j)$ restricting the chance of being performed by the same aircraft. In our numerical examples, we only consider the most necessary requirements which are the consistency between the arrival time/airport of the former flight $i$ and the departure time/airport of the latter flight $j$. $B$ denotes the set of airports to be considered in the model. $F_{e}^{t}$ denotes the set of flights for an aircraft $t$ in $T$ such that the flights in $F_{e}^{t}$ can be the last flight of aircraft $t$ since they 
terminate at the convenient airports in terms of maintenance feasibility. $F_{s}^{t}$ denotes the set of flights for an aircraft $t$ in $T$ such that the origin airport of flights in $F_{s}^{t}$ are the airport from which that aircraft starts the planning day. $P_{i}$ is the set of flights such that the passengers in flight $i$ might have connection.

For each $i \in F$ and $t \in T$, we redefine the fuel consumption as follows:

$$
F_{i}^{t}\left(f_{i}^{t}\right)= \begin{cases}\left(c_{1}^{i, t} \frac{1}{f_{i}^{t}}+c_{2}^{i, t} \frac{1}{\left(f_{i}^{t}\right)^{2}}+c_{3}^{i, t}\left(f_{i}^{t}\right)^{3}+c_{4}^{i, t}\left(f_{i}^{t}\right)^{2}\right) & \text { if }\left(y_{i}^{t}+\sum_{j \in U^{i}} x_{j i}^{t}\right)=1 \\ 0 & \text { if }\left(y_{i}^{t}+\sum_{j \in U^{i}} X_{j i}^{t}\right)=0\end{cases}
$$

so that if aircraft $t$ is not assigned to flight $i$, then $F_{i}^{t}\left(f_{i}^{t}\right)=0$.

The proposed nonlinear mixed integer programming model is provided below:

$$
\begin{aligned}
& \min \sum_{t \in T} \sum_{i \in F}\left(\sum_{j \in U^{i}} x_{j i}^{t}+y_{i}^{t}\right) \cdot \operatorname{Cspl}_{i} \cdot \max \left(0, \operatorname{Dem}_{i}-\operatorname{Cap}_{t}\right)+\sum_{i \in F} \sum_{t \in T}\left(c_{f u e l}+c_{\mathrm{CO}_{2}}\right) \cdot F_{i}^{t}\left(f_{i}^{t}\right)+\sum_{i \in F} \sum_{t \in T} y_{i}^{t} \cdot \text { Daily }_{t}+\sum_{i \in F} \sum_{t \in T} s_{i}^{t} \cdot \text { Idle }_{t} \\
& \text { s.t. } \sum_{j \in U^{i}} x_{j i}^{t}+y_{i}^{t}-\sum_{j \in D^{i}} x_{i j}^{t}-z_{i}^{t}=0 \quad \forall i \in F, t \in T \\
& \sum_{i \in F} y_{i}^{t} \leqslant 1 \quad \forall t \in T \\
& \sum_{t \in T}\left(y_{i}^{t}+\sum_{j \in U^{i}} x_{j i}^{t}\right)=1 \quad \forall i \in F \\
& \text { IF } \sum_{t \in T} x_{i j}^{t}=1 \\
& \text { THEN, } d_{j}-d_{i}-T A_{i j}^{t}-\sum_{t \in T} f_{i}^{t}-E\left[N C_{i}\right]-\sum_{t \in T} s_{i}^{t}=0 \quad \forall(i, j) \in A \\
& \operatorname{Pr}\left[N C_{i} \leqslant d_{j}-d_{i}-\sum_{t \in T} f_{i}^{t}-T P_{i j}\right] \geqslant \gamma_{i j} \quad \forall i \in F, j \in P_{i} \\
& \sum_{i \in F} f_{i}^{t} \leqslant \lambda_{t} \quad \forall t \in T \\
& \operatorname{IF}\left(y_{i}^{t}+\sum_{j \in U^{i}} x_{j i}^{t}\right)=1 \\
& \text { THEN, } f_{i}^{l} \leqslant f_{i}^{t} \leqslant f_{i}^{u} \forall i \in F, t \in T \\
& \text { ELSE } \\
& f_{i}^{t}=0 \\
& s_{i}^{t}=0 \\
& y_{i}^{t}=0 \quad \forall t \in T, i \in F \backslash F_{s}^{t} \\
& z_{i}^{t}=0 \quad \forall t \in T, i \in F \backslash F_{e}^{t} \\
& d_{i}^{l} \leqslant d_{i} \leqslant d_{i}^{u} \quad \forall i \in F \\
& s_{i}^{t} \geqslant 0 \quad \forall i \in F, t \in T \\
& x_{i j}^{t} \in\{0,1\} \quad \forall(i, j) \in A, t \in T \\
& y_{i}^{t} \in\{0,1\} \quad \forall i \in F, t \in T
\end{aligned}
$$

The objective function (3.2) is the sum of the spill cost, fuel consumption and $\mathrm{CO}_{2}$ emission cost, the daily aircraft usage cost and idle time cost, respectively. Constraint (3.3) is network balance equations. Constraints (3.4) and (3.5) are typical assignment constraints, such that each aircraft can be used for at most one flight sequence (string or path), and each flight can be performed by exactly one aircraft. Constraint (3.6) ensures that if two flights are performed by the same aircraft consecutively, time between the departures of these flights have to be greater than the sum of cruise time, non-cruise time and turnaround time. This time difference corresponds to idle time at the end of the first flight. Hence, when we add the idle time variable to (3.6), we obtain an equality constraint since the idle time is always nonnegative due to constraint (3.13). Constraint (3.7) is the chance constraint which ensures the service level of passengers' connection. This probabilistic constraint guarantees that the percentage of the passengers' connection satisfied between flights $(i, j)$ is greater than the minimum desired service level for each connecting flight pair, denoted as $\gamma_{i j}$, such that the probability of the time between arrival 
Table 4

Cost calculation for the proposed schedule.

\begin{tabular}{|c|c|c|c|c|c|}
\hline Tail\# & Flight \# & Fuel and $\mathrm{CO}_{2}$ & Idle time & Spill & Daily usage \\
\hline N3DUAA & $\begin{array}{l}2079 \\
1438 \\
1972\end{array}$ & $\begin{array}{l}5923.3 \\
5650 \\
2250.8\end{array}$ & $\begin{array}{l}1192.8 \\
- \\
-\end{array}$ & $\begin{array}{l}453.1 \\
431.5 \\
-\end{array}$ & 85,200 \\
\hline N412AA & $\begin{array}{l}2099 \\
1972 \\
2345 \\
2374\end{array}$ & $\begin{array}{l}4294.5 \\
3866.4 \\
2320.4 \\
1889.6\end{array}$ & $\begin{array}{l}- \\
- \\
-\end{array}$ & $\begin{array}{l}443.9 \\
693.6 \\
- \\
-\end{array}$ & 84,000 \\
\hline N4XGAA & $\begin{array}{r}2057 \\
2078 \\
346\end{array}$ & $\begin{array}{l}7035.2 \\
8260.5 \\
2994.3\end{array}$ & $\begin{array}{l}172.8 \\
- \\
-\end{array}$ & $\begin{array}{l}- \\
- \\
-\end{array}$ & 86,400 \\
\hline Total & & 44,485 & 1365.6 & 2022.1 & 255,600 \\
\hline
\end{tabular}

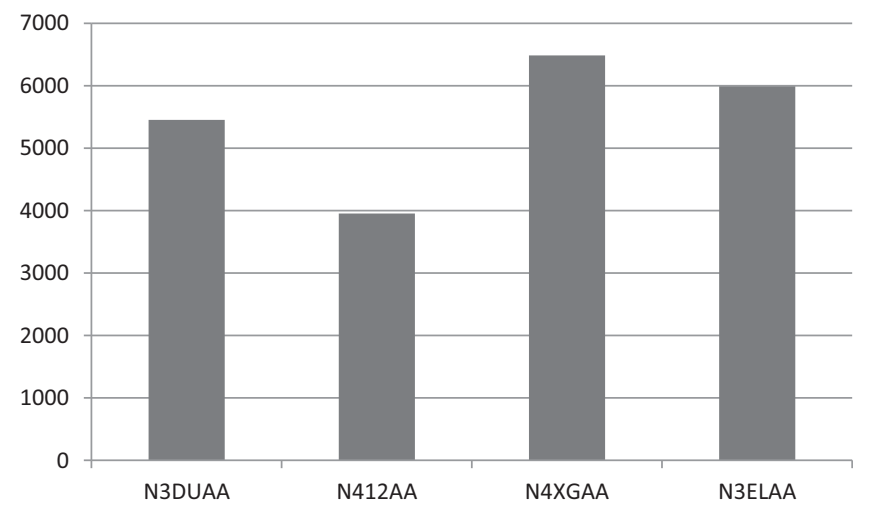

Fig. 2. The fuel and $\mathrm{CO}_{2}$ cost of flight 1438 with each aircraft type.

of flight $i$ and departure of flight $j$ being greater than the required connection time $T P_{i j}$ is at least $\gamma_{i j}$. Due to constraint sets (3.6) and (3.7), there is a critical tradeoff between the passenger connection service levels defined in the constraint set and the decision variables $s_{i}^{t}$ (the idle time of aircraft $t$ after flight $i$, i.e. slack time), which are penalized in the objective function of the proposed model. When $s_{i}^{t}$ values are increased, it is more likely to catch the connecting flight, i.e., higher passenger connection service levels. Constraint (3.8) ensures that total cruise time of an aircraft does not exceed a predetermined time limit in order to ensure maintenance feasibility. If a flight $i$ is performed by aircraft $t$ then constraint (3.9) limits cruise time change; cruise time of a flight cannot exceed the upper and lower bounds, else the corresponding variables $f_{i}^{t}$ and $s_{i}^{t}$ are set to zero. The aim of constraints (3.10) and (3.11) is to sustain maintenance policy intended in the published schedule, in this fashion first and last airport for each aircraft is determined. Constraint (3.12) sets the upper and lower bounds for departure time of each flight due to marketing requirements. As discussed above, it is guaranteed that the idle time is always nonnegative due to constraint (3.13). Constraints (3.14) and (3.15) guarantee that $x_{i j}^{t}$ and $y_{i}^{t}$ are binary variables and due to (3.3) all $z_{i}^{t}$ are also binary variables.

\subsection{Reformulation of the model}

In the proposed model, we have a nonlinear cost terms in the objective function and a probabilistic chance constraint. Therefore, we conduct a conic reformulation of the model so that we can utilize the recent advances in second order cone programming to solve the proposed formulation using a commercial solver.

In the objective function, the cost functions involve nonlinearity due to controllable cruise time associated with the changing the cruise speed of the aircraft. To handle nonlinearity, nonlinear mixed integer optimization often requires too much computation time. On the other hand, it may not result in exact solutions. In order to shorten the solution time and obtain an optimal solution, in this section we show the conic quadratic reformulation of the fuel consumption function $F_{i}^{t}\left(f_{i}^{t}\right)$ cost as discussed in Aktürk et al. (2009) and Günlük and Linderoth (2010). To simplify the presentation, we drop the indices of the variables and parameters.

$$
F(f)= \begin{cases}\left(c_{1} \frac{1}{f}+c_{2} \frac{1}{(f)^{2}}+c_{3}(f)^{3}+c_{4}(f)^{2}\right) & \text { if } w=1 \\ 0 & \text { if } w=0\end{cases}
$$

where $w=w_{i}^{t}=\left(y_{i}^{t}+\sum_{j \in U^{i}} x_{j i}^{t}\right)$, for simplicity. 
$F(f)$ is discontinuous and therefore its epigraph $E_{F}=\left\{(f, t) \in R^{2}: F(f) \leqslant t\right\}$ is non-convex. In the next proposition, we describe how the convexity of $E_{F}$ is obtained. A more detailed information can be found in Aktürk et al. (2009) and Günlük and Linderoth (2010).

Proposition 1. The convex hull of $E_{F}$ can be expressed as

$$
\begin{aligned}
& t \geqslant\left(c_{1} \cdot q+c_{2} \cdot \delta+c_{3} \cdot \phi+c_{4} \cdot v\right) \\
& w^{2} \leqslant q \cdot f \\
& w^{4} \leqslant f^{2} \cdot \delta \cdot 1 \\
& f^{4} \leqslant w^{2} \cdot \phi \cdot f \\
& f^{2} \leqslant v \cdot w
\end{aligned}
$$

in the constraint set. Moreover, each inequalities (3.16)-(3.20) can be represented by conic inequalities.

Proof. Perspective of a convex function $F(f)$ is $w F(f / w)$ (Hiriart-Urruty and Lemaréchal, 2001). Since each of the nonlinear terms $\frac{1}{f}, \frac{1}{f^{2}}, f^{3}$ and $f^{2}$ is a convex function for $f \geqslant 0$, then epigraph of the perspective of each term can be stated as $\frac{w^{2}}{f} \leqslant q, \frac{w^{4}}{f^{2}} \leqslant \delta, \frac{f^{3}}{w^{2}} \leqslant \phi$, and $\frac{f^{2}}{w} \leqslant v$, respectively, where $q, \delta, \phi$ and $v$ are the variables required for defining epigraphs. Since $w, f \geqslant 0$, they can be written as stated in the proposition.

Finally, observe that (3.17) and (3.20) are hyperbolic inequalities, (3.18) can be restated as two hyperbolic inequalities

$$
w^{2} \leqslant u \cdot f \text { and } u^{2} \leqslant \delta \cdot 1
$$

and (3.19) can be restated as

$$
f^{2} \leqslant u \cdot w \text { and } u^{2} \leqslant \phi \cdot f
$$

which can be written as a conic quadratic inequality.

We now have a linear fuel cost in the objective function with a set of conic inequalities given in (3.22)-(3.25) as in Aktürk et al. (2014). Furthermore, we take the flight time as the sum of cruise and non-cruise times. In our integrated model, the cruise time of each flight is a decision variable, whereas the non-cruise time of each flight $i \in F, N C_{i}$, is a given parameter. Arikan et al. (2013) performed an extensive study on airline flight schedules across several U.S. airlines and showed that the $\log$-Laplace distribution provides a good fit to the block time of a flight. Therefore, in our computational study, the non-cruise time of each flight, $N C_{i}$, is taken from a log-Laplace distribution. Consequently, probabilistic chance constraints could be represented in a closed form and written as second order conic inequalities in (3.26) as discussed in Duran et al. (2015). $F_{X}^{-1}\left(\gamma_{i j}\right)$ is the value of quantile function for log-Laplace distribution at $\gamma_{i j}$. After the reformulation, we could solve the following mathematical model using a commercial solver such as IBM ILOG CPLEX.

$$
\begin{array}{ll}
\min \quad & \sum_{t \in T} \sum_{i \in F}\left(\sum_{j \in U^{i}} x_{j i}^{t}+y_{i}^{t}\right) \cdot \operatorname{Cspl} l_{i} \cdot \max \left(0, \operatorname{Dem}_{i}-\operatorname{Cap}_{t}\right) \\
& +\sum_{i \in F} \sum_{t \in T}\left(c_{f u e l}+c_{\mathrm{CO}_{2}}\right) \cdot\left(c_{1}^{i, t} q_{i}^{t}+c_{2}^{i, t} \delta_{i}^{t}+c_{3}^{i, t} \phi_{i}^{t}+c_{4}^{i, t} v_{i}^{t}\right) \\
& +\sum_{i \in F} \sum_{t \in T} y_{i}^{t} \cdot \operatorname{Daily}_{t}+\sum_{i \in F} \sum_{t \in T} s_{i}^{t} \cdot I d l e_{t} \\
\text { s.to } \quad\left(y_{i}^{t}+\sum_{j \in U^{i}} x_{j i}^{t}\right)^{2} \leqslant q_{i}^{t} \cdot f_{i}^{t} \quad \forall i \in F, t \in T \\
\left(y_{i}^{t}+\sum_{j \in U^{i}} x_{j i}^{t}\right)^{4} \leqslant\left(f_{i}^{t}\right)^{2} \cdot \delta_{i}^{t} \cdot 1 \quad \forall i \in F, t \in T \\
\left(f_{i}^{t}\right)^{4} \leqslant\left(y_{i}^{t}+\sum_{j \in U^{i}} x_{j i}^{t}\right)^{2} \cdot \phi_{i}^{t} \cdot f_{i}^{t} \quad \forall i \in F, t \in T \\
\left(f_{i}^{t}\right)^{2} \leqslant v_{i}^{t} \cdot\left(y_{i}^{t}+\sum_{j \in U^{i}} x_{j i}^{t}\right)^{2} \quad \forall i \in F, t \in T \\
d_{j}-d_{i}-\sum_{t \in T} f_{i}^{t}-T P_{i j} \geqslant F_{X}^{-1}\left(\gamma_{i j}\right) \quad \forall i \in F, j \in P_{i} \\
(3.3)-(3.6),(3.8)-(3.15) \quad
\end{array}
$$




\section{Heuristic methods}

Although we could solve the small instances using the proposed integrated robust airline scheduling, aircraft fleeting and routing model, we could face some numerical stability problems due to the large variability of the problem parameters. Therefore, two heuristic methods are proposed to handle large size instances.

\subsection{Discretized approximation and cruise speed control algorithm}

To solve our problem faster than the integrated model, we propose discretized approximation model (hereafter DAM) which excludes nonlinearity caused by the cost function of fuel consumption and $\mathrm{CO}_{2}$ emission. In this algorithm, the controllable cruise times, which are defined by a continuous function in the integrated model, are approximated through a set of discrete cruise time settings.

DAM is a mixed integer programming model which solves robust airline scheduling, aircraft fleeting and routing problems simultaneously. As distinct from the integrated model, in DAM, cruise times can only take a predetermined value from an interval rather than any value from that interval. For example, while cruise time of a flight in the integrated model can take any value between 85 and $115 \mathrm{~min}$, in DAM the cruise time can be in increments of five minutes such as 85, 90, 95, 100, 105,110 and 115 . In this way, instead of a nonlinear cost function, we calculate the price of each cruise time option and use linear terms to denote the fuel consumption and $\mathrm{CO}_{2}$ emission costs in the objective function. Consequently, DAM can be solved faster than the integrated model.

In order to adapt these changes, we introduce the following additional parameters and a set of binary variables for each flight and aircraft:

$c r s_{i k}^{t}$ : The $k$ th cruise time option of flight $i$ when it is performed by aircraft $t$

$\operatorname{cost}_{i k}^{t}$ : The cost of $\operatorname{crs}_{i k}^{t}$ which is equal to $\left(c_{f u e l}+c_{\mathrm{CO}_{2}}\right) \cdot F\left(\right.$ crs $\left._{i k}^{t}\right)$

$\sigma_{i k}^{t}: 1$ if cruise time of flight $i$ takes the $k$ th setting for aircraft $t$ and is 0 ow.

The formulation of DAM is as follows:

$$
\begin{array}{ll}
\min & \sum_{t \in T} \sum_{i \in F}\left(\sum_{j \in U^{i}} x_{j i}^{t}+y_{i}^{t}\right) \cdot \operatorname{Cspl}_{i} \cdot \max \left(0, \text { Dem }_{i}-\operatorname{Cap}_{t}\right)+ \\
& \sum_{i \in F} \sum_{t \in T} \sum_{k=1}^{p} \sigma_{i k}^{t} \cdot \operatorname{cost}_{i k}^{t}+\sum_{i \in F} \sum_{t \in T} y_{i}^{t} \cdot \text { Daily }_{t}+\sum_{i \in F} \sum_{t \in T} s_{i}^{t} \cdot \text { Idle }_{t} \\
\text { s. to } & \sum_{k=1}^{p} \sigma_{i k}^{t}=\left(y_{i}^{t}+\sum_{j \in U^{i}} x_{j i}^{t}\right) \quad \forall i \in F, t \in T \\
& \sum_{k=1}^{p} \sigma_{i k}^{t} \cdot \operatorname{crs}_{i k}^{t}=f_{i}^{t} \quad \forall i \in F, t \in T \\
\text { (3.3)-(3.6), (3.8)-(3.15), (3.26) }
\end{array}
$$

In DAM, the objective function is the sum of spill cost, the costs of fuel consumption and $\mathrm{CO}_{2}$ emission, daily usage cost and idle time cost. As different from the integrated model, the cost of fuel consumption and $\mathrm{CO}_{2}$ emission is represented by the linear term $\sum_{i \in F} \sum_{t \in T} \sum_{k=1}^{p} \sigma_{i k}^{t} \cdot \cos _{i k}^{t}$. Constraints (4.2) and (4.3) ensure that when flight $i$ is performed by aircraft $t$, the cruise time of that flight takes one of the discrete cruise time settings. The remaining constraints are same as with the integrated model.

We could solve the robust airline scheduling, aircraft fleeting and routing problem with DAM in a reasonable computation time. However, since we have discretized cruise time in DAM, there is still a chance to improve that solution by considering a continuous value of cruise time. Therefore, after fixing fleeting and routing with DAM, in order to decide continuous values of cruise time, we solve the cruise speed control model (hereafter CSCM), which is suggested by Duran et al. (2015). CSCM is a nonlinear second order cone programming model which solves robust airline scheduling problem by considering departure timing, cruise time control and idle time insertion. Since the fleeting and routing decisions are made by DAM, CSCM only deals with continuous decision variables. Therefore, even if it is a nonlinear model it can still be solved faster than the integrated model.

In order to use the fleeting and routing generated by DAM as parameters of CSCM, we introduce the following additional notation:

$\bar{w}_{i}^{t}$ : A binary parameter that is 1 if flight $i$ is performed by aircraft $t$ in the solution of DAM

$\bar{A}$ : Set of flights $(i, j)$ such that $(i, j)$ are two consecutive flights performed by the same aircraft in DAM 
The formulation of CSCM is as follows:

$$
\begin{aligned}
& \min \sum_{i \in F} \sum_{t \in T} \bar{w}_{i}^{t} \cdot\left(\left(c_{\text {fuel }}+c_{\mathrm{CO}_{2}}\right) F\left(f_{i}^{t}\right)+s_{i}^{t} \cdot \text { Idle }_{t}\right) \\
& \text { s.to } x_{j}-x_{i}-T A_{i j}-\sum_{t \in T} \bar{w}_{i}^{t} \cdot f_{i}^{t}-E\left[A_{i}\right]-\sum_{t \in T} \bar{w}_{i}^{t} \cdot s_{i}^{t}=0 \quad \forall(i, j) \in \bar{A} \\
& f_{i}^{l} \cdot \bar{w}_{i}^{t} \leqslant f_{i}^{t} \leqslant f_{i}^{u} \cdot \bar{w}_{i}^{t} \quad \forall i \in F, t \in T \\
& s_{i}^{t} \leqslant M \cdot \bar{w}_{i}^{t} \quad \forall i \in F, t \in T \\
& \quad \text { (3.8), (3.12), (3.13), (3.26) }
\end{aligned}
$$

In the objective function of CSCM, the costs of idle time insertion, fuel consumption and $\mathrm{CO}_{2}$ emission are minimized. Constraint (4.5) ensures the aircraft connection for the routing generated by DAM. If aircraft $t$ performs flight $i$ as a result of DAM, constraint (4.6) keeps the cruise time of each flight between the upper and lower limits, otherwise $f_{i}^{t}$ value is set to zero. Similarly, if aircraft $t$ performs flight $i$ as a result of DAM, then $s_{i}^{t}$ can be nonnegative, otherwise it is set to zero by constraint (4.7).

In discretized approximation and cruise speed control algorithm (hereafter Heuristic 1), we solve two models sequentially. In Fig. 3, we illustrate the flow chart of the algorithm. Initially we solve the DAM and fix the fleeting and routing decision, then we solve the CSCM in order to find the minimum sum of idle time, fuel consumption and $\mathrm{CO}_{2}$ emission costs, e.g. corresponding cruise time, for a given fleeting and routing decision. In this way, instead of solving the integrated model, which is a nonlinear MIP model; as a heuristic method we propose to solve first a MIP model, e.g., DAM, and then a nonlinear model, CSCM, so that total solution time is quite smaller than the integrated model as discussed earlier.

\subsection{Multi-stage triplet search algorithm}

As another observation, when we remove the cost terms related to the continuous decision variables from the objective function of the integrated model, we observe that solution time decreases drastically. This is due to two reasons. First, by removing fuel consumption and $\mathrm{CO}_{2}$ emission costs, we exclude nonlinearity and get a MIP model as in the case of DAM. Second, when we remove the cost term of the idle time insertion as well as the cost term related to fuel consumption, aircraft fleeting and routing decisions are given more easily. To elaborate, without idle time, fuel consumption and $\mathrm{CO}_{2}$ emission costs, the model only considers the tradeoff between daily usage and spill cost so it neglects the costs of idle time insertion and cruise time change. Therefore, we introduce daily usage and spill costs model (hereafter DSCM) in which the idle time insertion, fuel consumption and $\mathrm{CO}_{2}$ emission costs are removed. All constraints of the integrated model in Section 4.1 are valid in DSCM, however the cost terms $\sum_{i \in F} \sum_{t \in T}\left(c_{f u e l}+c_{\mathrm{CO}_{2}}\right) \cdot F_{i}^{t}\left(f_{i}^{t}\right)$ and $\sum_{i \in F} \sum_{t \in T} s_{i}^{t} \cdot I_{d l e_{t}}$ are extracted from the objective function.

Although we get a non-dominated solution regarding daily usage and spill costs by DSCM, the generated solutions might have high cost of idle time and fuel consumption since DSCM takes idle time and fuel as free. In order to enhance a solution generated by DSCM, the cost of fuel and idle time should be taken into account. Thus, we take the solution of DSCM and then by fixing fleeting and routing of DSCM, we solve CSCM which is presented in the previous section.

Even if solving CSCM after DSCM can improve the cost of idle time and fuel, this improvement is local since CSCM is solved within the fixed fleeting and routing of DSCM. By driving DSCM to generate fleeting and routing which are also favorable regarding the costs of idle time and fuel, the sequential solution of DSCM and CSCM can find better solutions. For this

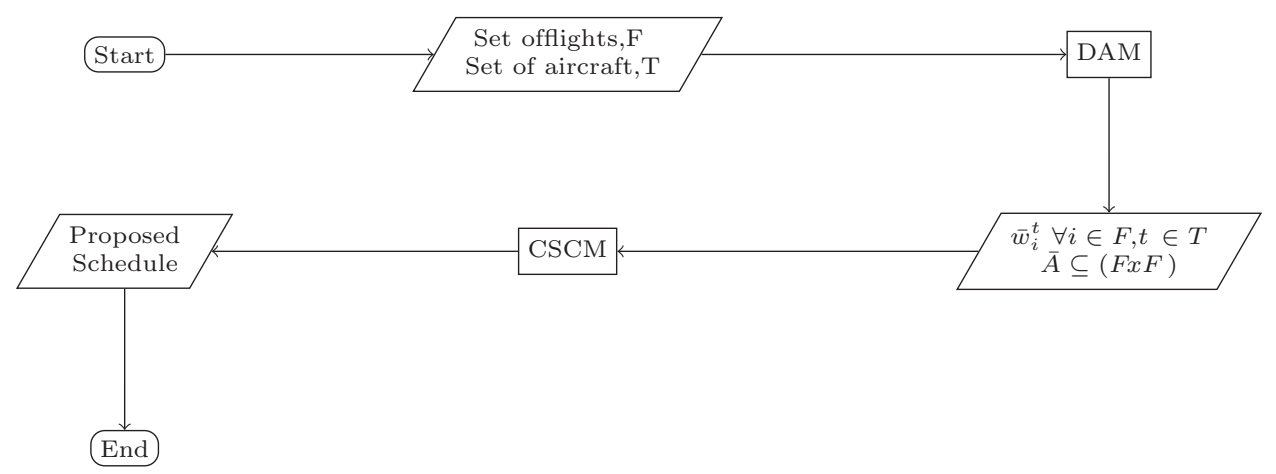

Fig. 3. Discretized approximation and cruise speed control algorithm. 
purpose, initially we introduce triplet which is a constituent of a fleeting and routing in an airline schedule, then present a search algorithm over triplets to improve the solutions generated by the sequential solution of DSCM and CSCM.

Definition 1. A triplet, $(i, j, t)$ is a collection of two consecutive flights $i$ and $j$ and the aircraft $t$ which performs them. A triplet has the cruise times and idle time information regarding the flights in the triplet.

The cost of a triplet is calculated as follows: the fuel consumption and $\mathrm{CO}_{2}$ emission costs are calculated for two flights and the idle time cost of the aircraft between these flights are summed and then the minimum required fuel consumption and $\mathrm{CO}_{2}$ emission costs for these flights are extracted. The minimum required fuel consumption and $\mathrm{CO}_{2}$ emission costs are calculated by assuming these flights are performed by the most efficient aircraft in the set of aircraft at optimum speed. Therefore, the cost of each triplet can be thought as the improvement capacity on the cost of that triplet.

In DSCM, each decision variable $x_{i j}^{t}$ refers to a triplet so by fixing $x_{i j}^{t}=0$ beforehand, we avoid DSCM to have triplet $(i, j, t)$ in the generated solution. In this manner, we present multi-stage triplet search algorithm in which we employ a variablefixing idea, and solve DSCM and CSCM models sequentially.

Algorithm 1: Two Stage Algorithm

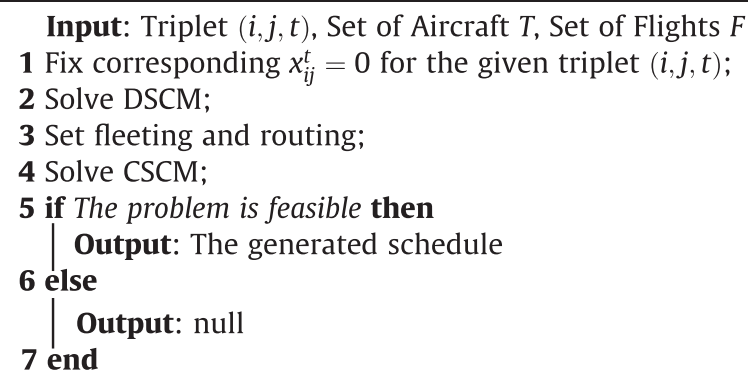

Heuristic 2: Multi-Stage Triplet Search Algorithm

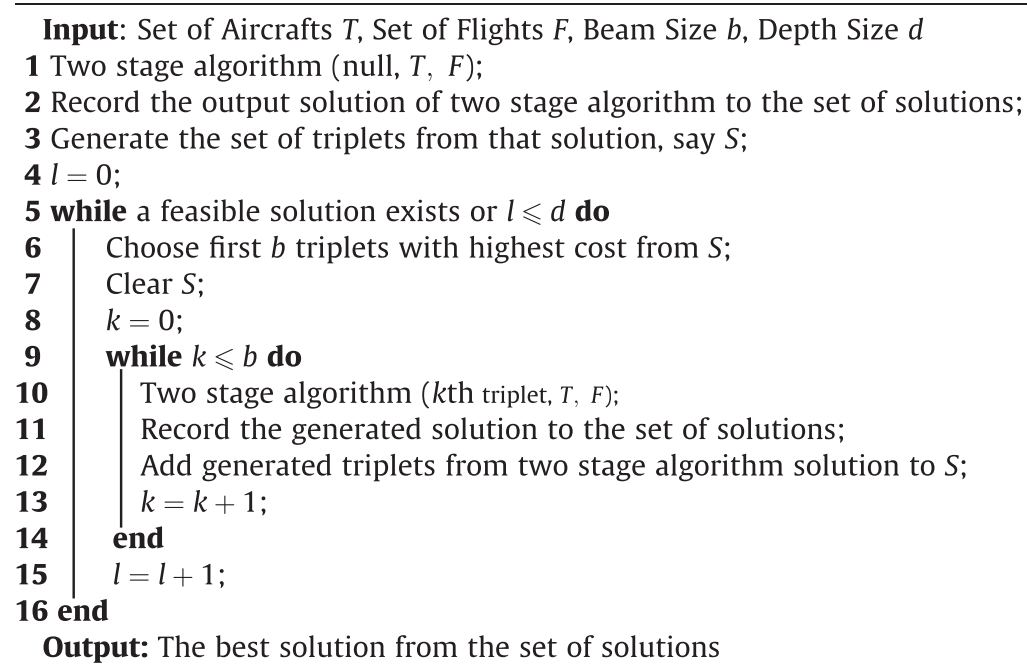

The multi-stage triplet search algorithm, as illustrated in Heuristic 2, takes a set of aircraft and a set of flights with given beam size $b$ and depth size $d$ as an input. As an initialization step at the root node, we apply two stage algorithm, given in Algorithm 1, without a triplet. Afterwards, the set of triplets, $S$, is generated from the root node solution. After initialization step, a procedure which repeats itself for each depth starts. At the beginning of each depth, we have a set of triplets coming from the previous step, $S$, note that for the first depth, set of triplets comes from the root node solution. From $S$, we choose first $b$ triplets with highest cost and then remove them from the solution set. For each chosen triplet, $b$ times at total for a depth, we apply two stage algorithm to get a solution and add the generated triplets to $S$ for the next depth. Since we apply this procedure $d$ times, we get one solution from the root node and $b$ solutions at each depth. In sum, we eventually report the best solution among $b \cdot d+1$ feasible solutions generated by the multi-stage triplet search algorithm. 


\section{Computational study}

In this section, we compare the performances of three solution methods which are the integrated approach and two heuristics, in terms of different airline cost components against the published schedule along with the required CPU times. In this computational study, there are four experimental factors, and their corresponding levels are given in Table 5.

The first factor is the fuel cost, which is the price of jet fuel per ton. The fuel prices are taken as \$1.8/gallon for the lower setting and $\$ 3.6$ gallon for the higher setting, or accordingly $\$ 600 /$ ton and $\$ 1200 /$ ton, respectively. Another important factor is the opportunity cost of spilled passengers of flight $i, \operatorname{Cspl}_{i}$. We again consider two different levels of base spill cost, denoted as Factor B. The base spill cost is used as a multiplier to calculate the spill cost of a flight per passenger as discussed in a recent study conducted by Duran et al. (2015). One of the main contributions of the integrated approach is to improve the aircraft utilization by solving the aircraft scheduling, routing and fleeting problems simultaneously along with the cruise speed control decisions. On the other hand, there is a significant tradeoff between the aircraft utilization and overall operational expenses, such as fuel and carbon emission costs. Due to a possible increase in the utilization of a fuel efficient aircraft, total fuel consumption cost could be decreased for the entire flight network in albeit of additional spill costs. Consequently, we need to quantify the aircraft utilization along with the operational expenses in our objective function. We have used the parameter Idle $e_{t}$ for each aircraft type $t$ as a surrogate parameter to evaluate a possible tradeoff between the aforementioned aircraft utilization and operational expenses in our proposed mathematical model. Since it is difficult to accurately estimate these parameters, we have taken the daily usage cost of each aircraft type, Daily $y_{t}$ as one of the experimental factors. The third experimental factor, $v$, is used for the calculation of the daily usage cost of an aircraft. In our study, we consider the daily usage cost of an aircraft which refers to the sum of the fixed operating costs and the lost opportunity cost when that aircraft is used as discussed in Section 2. We calculate daily usage cost related to the unit idle time cost since it is rational that a valuable aircraft has both high unit idle time cost and daily usage cost. For each aircraft type, we set its daily usage cost to low and high levels, respectively, to observe its impact on the overall results as follows.

$$
\text { Daily }_{t}=v \cdot \text { Idle }_{t} \cdot 60
$$

The last factor is the minimum desired service level of the passengers' connections. As distinct from the first three factors, this factor has three levels. While the first three factors have linear effects on the objective function, the effect of service level on the objective function is not as straightforward as the other factors since it is not a direct cost term of the objective function. Therefore, in order to reveal its effect in the experimental design, we choose to adopt three levels on this factor.

In this study, we use the flight network, which is in the work of Aktürk et al. (2014). The published schedule of a major U. S. airline is provided in Table 6. The flight information were taken from the Bureau of Transportation Statistics (BTS) database (BTS, 2010). In the published schedule, each column represents the tail number, flight number, departure and arrival airport, departure time, flight block time and arrival time of each flight respectively. From the published schedule, we take the set of flights and set of aircraft as input in our model.

Among these 114 flights, we generate three different flight networks. The first one is 23 flight network; the second is 35 flight network and the last one is 114 flight network as given in Table 6 . We conduct all computational study over these flight networks. Furthermore, we have taken 3 replications for each factor combination, e.g. out of $2 \cdot 2 \cdot 2 \cdot 3$ combinations in a full-factorial experimental design, resulting in 72 randomly generated runs for each flight network.

\subsection{Analysis on the integrated model}

We start the analysis on the results of our computational study by pointing out the significance of our integrated approach. For this purpose, we compare the cost of the published schedule, which is generated by the sequential approach, and the proposed schedule, which is the output of our proposed integrated robust airline scheduling, aircraft fleeting and routing model with cruise speed control.

We first analyze the 23 flight network in terms of operational efficiency. In particular, this small network consists of 23 flights that are performed by seven aircraft. The tail numbers of these aircraft are N3DUAA, N5DXAA, N412AA, N531AA, N4XGAA, N544AA and N3ELAA. The corresponding flights are available in Table 6 . In this schedule, the average number

Table 5

Factor values.

\begin{tabular}{lllll}
\hline Factors & Description & Levels & Medium(1) & High(2) \\
\cline { 3 - 5 } & & Low $(0)$ & - & $\$ 1200$ \\
\hline A & Fuel cost & $\$ 600$ & - & \\
B & Base spill cost & $\$ 15$ & - & 10 \\
C & $v$ & 1 & $85 \%$ & $95 \%$ \\
D & Service level & $75 \%$ & \\
\hline
\end{tabular}


Table 6

Published schedule for 114 flight network.

\begin{tabular}{|c|c|c|c|c|c|c|c|c|c|c|c|}
\hline Tail \# & Flight \# & From & To & Dep. time & Dur. & Tail \# & Flight \# & From & To & Dep. time & Dur. \\
\hline \multirow[t]{4}{*}{ N530AA } & 398 & ORD & LGA & $06: 15$ & 134 & N3ETAA & 1704 & ORD & EWR & 06:35 & 125 \\
\hline & 319 & LGA & ORD & $09: 25$ & 170 & & 1883 & EWR & ORD & $09: 30$ & 160 \\
\hline & 2329 & ORD & DFW & $13: 35$ & 155 & & 810 & ORD & DCA & $13: 10$ & 105 \\
\hline & 2364 & DFW & ORD & 17:00 & 150 & & 2013 & DCA & ORD & $15: 45$ & 135 \\
\hline \multirow[t]{4}{*}{ N459AA } & 394 & ORD & LGA & $06: 50$ & 135 & & 2013 & ORD & LAS & 19:00 & 250 \\
\hline & 321 & LGA & ORD & $10: 00$ & 170 & N3DYAA & 1063 & ORD & LAX & $08: 50$ & 275 \\
\hline & 366 & ORD & LGA & $13: 55$ & 140 & & 874 & LAX & ORD & $14: 30$ & 255 \\
\hline & 347 & LGA & ORD & $17: 15$ & 170 & & 874 & ORD & BOS & $19: 45$ & 135 \\
\hline \multirow[t]{5}{*}{ N531AA } & 2303 & ORD & DFW & $06: 45$ & 155 & N3DRAA & 1021 & ORD & LAS & $08: 30$ & 245 \\
\hline & 2336 & DFW & ORD & $10: 10$ & 140 & & 1544 & LAS & ORD & $13: 25$ & 215 \\
\hline & 1053 & ORD & AUS & $13: 25$ & 170 & & 1544 & ORD & DCA & $18: 00$ & 105 \\
\hline & 336 & AUS & ORD & 17:00 & 165 & N5DXAA & 1048 & ORD & MIA & $07: 35$ & 190 \\
\hline & 336 & ORD & LGA & $20: 40$ & 125 & & 1763 & MIA & ORD & $11: 55$ & 200 \\
\hline \multirow[t]{3}{*}{ N4XGAA } & 2079 & ORD & SAN & $08: 45$ & 270 & & 1899 & ORD & MIA & $16: 20$ & 185 \\
\hline & 1438 & SAN & ORD & $14: 00$ & 250 & N454AA & 2441 & ORD & ATL & $06: 30$ & 120 \\
\hline & 346 & ORD & LGA & $19: 50$ & 135 & & 1986 & ATL & ORD & 09:15 & 135 \\
\hline \multirow[t]{3}{*}{ N598AA } & 1341 & ORD & SFO & $07: 50$ & 295 & & 1872 & ORD & $\mathrm{MCO}$ & $12: 25$ & 160 \\
\hline & 348 & SFO & ORD & $13: 30$ & 265 & & 1131 & MCO & ORD & $15: 50$ & 185 \\
\hline & 1521 & ORD & TUS & $19: 15$ & 235 & N4YMAA & 1137 & ORD & MSY & $08: 20$ & 145 \\
\hline \multirow{4}{*}{ N439AA } & 2455 & ORD & PHX & $07: 10$ & 240 & & 1768 & MSY & ORD & $11: 30$ & 150 \\
\hline & 358 & PHX & ORD & $11: 55$ & 210 & & 1768 & ORD & PHL & $15: 05$ & 125 \\
\hline & 358 & ORD & LGA & $16: 25$ & 145 & & 1697 & PHL & ORD & $18: 00$ & 155 \\
\hline & 371 & LGA & ORD & $20: 00$ & 155 & N467AA & 1823 & ORD & PBI & $09: 20$ & 175 \\
\hline \multirow[t]{5}{*}{ N475AA } & 407 & ORD & STL & $06: 20$ & 70 & & 2067 & PBI & ORD & $13: 00$ & 200 \\
\hline & 755 & STL & ORD & $08: 35$ & 75 & & 2067 & ORD & STL & $17: 15$ & 70 \\
\hline & 755 & ORD & SAT & $10: 45$ & 180 & & 1186 & STL & ORD & $19: 10$ & 80 \\
\hline & 408 & SAT & ORD & $14: 30$ & 160 & N536AA & 2305 & ORD & DFW & $07: 45$ & 160 \\
\hline & 408 & ORD & PHL & $18: 05$ & 125 & & 2344 & DFW & ORD & $11: 35$ & 140 \\
\hline \multirow[t]{4}{*}{ N3EEAA } & 876 & ORD & BOS & $06: 35$ & 130 & & 1201 & ORD & STL & $14: 50$ & 65 \\
\hline & 413 & BOS & ORD & 09:35 & 185 & & 1815 & STL & ORD & $17: 00$ & 80 \\
\hline & 413 & ORD & SNA & $13: 45$ & 275 & & 1815 & ORD & SLC & $19: 15$ & 270 \\
\hline & 1262 & SNA & ORD & $19: 10$ & 230 & N420AA & 1686 & ORD & RDU & $06: 50$ & 110 \\
\hline \multirow[t]{2}{*}{ N4YDAA } & 451 & ORD & SFO & $09: 45$ & 295 & & 2435 & RDU & ORD & 09:25 & 135 \\
\hline & 554 & SFO & ORD & $15: 45$ & 265 & & 2435 & ORD & PHX & $12: 35$ & 235 \\
\hline \multirow[t]{4}{*}{ N3ERAA } & 496 & ORD & DCA & $06: 45$ & 100 & & 1206 & PHX & ORD & $17: 15$ & 205 \\
\hline & 1715 & DCA & ORD & $09: 15$ & 130 & N546AA & 1462 & ORD & EWR & $08: 00$ & 140 \\
\hline & 1715 & ORD & LAS & $12: 25$ & 255 & & 1387 & EWR & ORD & $11: 25$ & 160 \\
\hline & 1708 & LAS & ORD & $17: 20$ & 220 & & 1397 & ORD & $\mathrm{MCO}$ & $15: 00$ & 160 \\
\hline \multirow[t]{3}{*}{ N5CLAA } & 1425 & ORD & SNA & $08: 25$ & 280 & & 1221 & MCO & ORD & $18: 25$ & 175 \\
\hline & 556 & SNA & ORD & $14: 00$ & 240 & N4WPAA & 2311 & ORD & DFW & 09:05 & 155 \\
\hline & 1940 & ORD & MIA & 19:25 & 180 & & 2348 & DFW & ORD & $12: 35$ & 140 \\
\hline \multirow[t]{4}{*}{ N535AA } & 2460 & ORD & RSW & $06: 45$ & 165 & & 1797 & ORD & STL & $15: 50$ & 70 \\
\hline & 564 & RSW & ORD & $10: 20$ & 185 & & 1982 & STL & ORD & $18: 00$ & 80 \\
\hline & 1446 & ORD & EWR & $14: 55$ & 165 & & 1339 & ORD & SAN & $20: 15$ & 270 \\
\hline & 1411 & EWR & ORD & $18: 45$ & 165 & N5EBAA & 2375 & ORD & EGE & $08: 10$ & 175 \\
\hline \multirow[t]{3}{*}{ N3DMAA } & 568 & ORD & FLL & $07: 25$ & 175 & & 2378 & EGE & ORD & $12: 25$ & 165 \\
\hline & 711 & FLL & ORD & $11: 10$ & 195 & & 1677 & ORD & SNA & $18: 40$ & 270 \\
\hline & 2021 & ORD & SJU & $15: 25$ & 275 & N3DUAA & 2099 & ORD & LAX & 07:00 & 270 \\
\hline \multirow[t]{5}{*}{ N544AA } & 2463 & ORD & $\mathrm{MCI}$ & $06: 25$ & 90 & & 1972 & LAX & ORD & $12: 40$ & 245 \\
\hline & 754 & $\mathrm{MCI}$ & ORD & $08: 40$ & 90 & & 1972 & ORD & RDU & $17: 45$ & 115 \\
\hline & 2321 & ORD & DFW & $11: 15$ & 155 & N3ELAA & 2057 & ORD & SJU & $08: 30$ & 290 \\
\hline & 2356 & DFW & ORD & $14: 40$ & 140 & & 2078 & SJU & ORD & $14: 25$ & 335 \\
\hline & 2487 & ORD & DEN & $17: 50$ & 165 & N3DTAA & 2363 & ORD & HDN & 09:50 & 170 \\
\hline N3EBAA & 1565 & ORD & MSP & $06: 40$ & 90 & & 2318 & HDN & ORD & $13: 40$ & 170 \\
\hline & 779 & MSP & ORD & 09:00 & 85 & N412AA & 2345 & ORD & DFW & $17: 15$ & 155 \\
\hline & 779 & ORD & SAN & 11:35 & 260 & & 2374 & DFW & ORD & $20: 40$ & 130 \\
\hline & 1358 & SAN & ORD & $16: 45$ & 235 & & & & & & \\
\hline & 1358 & ORD & BOS & $21: 50$ & 125 & & & & & & \\
\hline
\end{tabular}

of flights operated by a single aircraft is approximately 3 . The average utilization of these aircraft is around $73 \%$. Note that we consider the utilization of an aircraft as the ratio of the total time spent in the air, i.e., total cruise time to the total available cruise time for that aircraft denoted by $\lambda_{t}$ in our problem formulation. 
We next track the changes of the proposed model at these performance measures in order to interpret the impact of the integrated approach as well as the cruise time flexibility. Table 7 illustrates the values for these measures of the proposed schedule with respect to the different factor values. As the results over all instances row indicates that the integrated model yields higher aircraft utilization while decreasing the total number of aircraft required to perform these flights. Moreover, we provide the average cruise time change per flight because it is worth to note that when the daily usage cost factor is high (i.e, $C=2$ ), the model achieves to decrease the total number of aircraft in all instances by controlling cruise time more aggressively.

It is clear that the integration and cruise time change increase the efficiency of the flight schedule in comparison to sequential approach however it is intricate to evaluate how valuable this increase because of the network effect and the nontrivial cost function of the fuel consumption. As a next step, we provide how these changes correspond to cost improvement. We calculate the cost improvement for total cost and other cost terms for each factor setting as follows:

$$
\text { Improvement }=\frac{\text { Cost of Alternative }- \text { Cost of Our Model }}{\text { Cost of Alternative }}
$$

As it is shown in Table 8, our integrated model provides around 17\% average improvement on the total cost of the published schedule over all factor levels. Moreover, from Table 8, the tradeoff between daily usage cost and fuel consumption and $\mathrm{CO}_{2}$ emission costs can be deduced. When daily usage cost is high, the improvement over the fuel and $\mathrm{CO}_{2}$ emission costs is limited around $4 \%$ at maximum and the cost improvement over daily usage cost is around $14 \%$ at minimum. That means in order to decrease total number of aircraft used, the model compresses the cruise time of flights in albeit of additional fuel consumption. Similarly, when the cost of fuel consumption and $\mathrm{CO}_{2}$ emission is high, the average improvement on the daily usage cost decreases from $9 \%$ to $7 \%$ approximately. This is an important advantage of the proposed model, since we could now evaluate a possible tradeoff between the number of aircraft needed to cover all flights and the corresponding overall operational expenses.

\subsubsection{Scenario analysis}

The extensive computational study results intrigue several questions on model behavior and its performance. In this part, some insights into model dynamics are provided by realizing different scenarios.

Table 7

Efficiency of the proposed model.

\begin{tabular}{|c|c|c|c|c|c|c|c|c|c|c|c|c|c|}
\hline & & \multicolumn{3}{|c|}{ Avg. aircraft util. (\%) } & \multicolumn{3}{|c|}{ Avg. \# of flights per route } & \multicolumn{3}{|c|}{$\begin{array}{l}\text { Avg. cruise time change per } \\
\text { flight }(\%)\end{array}$} & \multicolumn{3}{|c|}{ \# of aircraft used } \\
\hline & & Min. & Avg. & Max. & Min. & Avg. & Max. & Min. & Avg. & Max. & Min. & Avg. & Max. \\
\hline \multirow[t]{2}{*}{ A } & 0 & 72 & 79 & 84 & 3.29 & 3.61 & 3.83 & 0.82 & 1 & 1.30 & 6 & 6.42 & 7 \\
\hline & 2 & 72 & 78 & 84 & 3.29 & 3.56 & 3.83 & 0.83 & 1 & 1.09 & 6 & 6.5 & 7 \\
\hline \multirow[t]{2}{*}{ B } & 0 & 72 & 78 & 84 & 3.29 & 3.56 & 3.83 & 0.95 & 1.02 & 1.30 & 6 & 6.5 & 7 \\
\hline & 2 & 72 & 79 & 84 & 3.29 & 3.61 & 3.83 & 0.82 & 0.98 & 1.09 & 6 & 6.42 & 7 \\
\hline \multirow[t]{2}{*}{ C } & 0 & 72 & 73 & 84 & 3.29 & 3.33 & 3.83 & 0.82 & 0.94 & 1.06 & 6 & 6.92 & 7 \\
\hline & 2 & 84 & 84 & 84 & 3.83 & 3.83 & 3.83 & 1.04 & 1.06 & 1.3 & 6 & 6 & 6 \\
\hline \multirow[t]{3}{*}{$\mathrm{D}$} & 0 & 72 & 79 & 84 & 3.29 & 3.58 & 3.83 & 0.82 & 1.01 & 1.09 & 6 & 6.46 & 7 \\
\hline & 1 & 72 & 79 & 84 & 3.29 & 3.58 & 3.83 & 0.82 & 1.01 & 1.30 & 6 & 6.46 & 7 \\
\hline & 2 & 72 & 79 & 84 & 3.29 & 3.58 & 3.83 & 0.82 & 0.99 & 1.05 & 6 & 6.46 & 7 \\
\hline \multicolumn{2}{|c|}{ All ins. } & 72 & 79 & 84 & 3.29 & 3.58 & 3.83 & 0.82 & 1 & 1.30 & 6 & 6.46 & 7 \\
\hline
\end{tabular}

Table 8

Cost improvement over the published schedule.

\begin{tabular}{|c|c|c|c|c|c|c|c|c|c|c|c|c|c|}
\hline & & \multicolumn{3}{|c|}{ Fuel and $\mathrm{CO}_{2}(\%)$} & \multicolumn{3}{|c|}{ Idle time (\%) } & \multicolumn{3}{|c|}{ Daily usage (\%) } & \multicolumn{3}{|c|}{ Total (\%) } \\
\hline & & Min. & Avg. & Max. & Min. & Avg. & Max. & Min. & Avg. & Max. & Min. & Avg. & Max. \\
\hline \multirow[t]{2}{*}{ A } & 0 & 4.2 & 8.3 & 14.5 & 89.3 & 93.8 & 96 & 0 & 8.6 & 14.7 & 14.9 & 18 & 22.8 \\
\hline & 2 & 4.2 & 9.8 & 14.5 & 89.3 & 93.5 & 95.7 & 0 & 7.4 & 14.7 & 13.4 & 16 & 19.8 \\
\hline \multirow[t]{2}{*}{ B } & 0 & 4.2 & 9.3 & 14.5 & 89.3 & 93.4 & 95.7 & 0 & 7.4 & 14.7 & 14.5 & 17.9 & 22.8 \\
\hline & 2 & 4.2 & 8.2 & 14.5 & 89.3 & 93.8 & 96 & 0 & 8.6 & 14.7 & 13.4 & 16 & 21.2 \\
\hline \multirow[t]{2}{*}{ C } & 0 & 4.2 & 13.4 & 14.5 & 89.2 & 93.1 & 96 & 0 & 1.23 & 14.7 & 13.7 & 18.9 & 22.8 \\
\hline & 2 & 4.2 & 4.2 & 4.2 & 91.8 & 94 & 95.7 & 14.7 & 14.7 & 14.7 & 13.4 & 15.1 & 16.6 \\
\hline \multirow[t]{3}{*}{ D } & 0 & 4.2 & 8.8 & 14.5 & 92.2 & 94.6 & 96 & 0 & 8 & 14.7 & 13.5 & 17.1 & 22.8 \\
\hline & 1 & 4.2 & 8.8 & 14.5 & 91.3 & 93.9 & 95.4 & 0 & 8 & 14.7 & 13.4 & 17 & 22.6 \\
\hline & 2 & 4.2 & 8.8 & 14.5 & 89.3 & 92.3 & 93.8 & 0 & 8 & 14.7 & 13.4 & 16.9 & 22.3 \\
\hline \multicolumn{2}{|c|}{ All ins. } & 4.2 & 8.8 & 14.5 & 89.3 & 93.6 & 96 & 0 & 8 & 14.7 & 13.4 & 17 & 22.8 \\
\hline
\end{tabular}


What if cruise time change is not allowed?

We continue the analysis on the results of our computational study by indicating what would happen without cruise time control. By this means, we present the analysis on the integrated model and compare its performance with other integrated models, which do not consider cruise speed control. For a fixed cruise time setting, we also consider two models. Both models are integrated ones such that airline scheduling, aircraft fleeting and routing decisions are made within the consideration of passengers' service level and maintenance requirements. The first model tries to minimize the sum of spill cost, fuel consumption and $\mathrm{CO}_{2}$ emission cost, idle time cost and daily usage cost without cruise time control. The second one tries to minimize the sum of spill cost, idle time cost and daily usage cost but considers neither fuel consumption nor $\mathrm{CO}_{2}$ emissions cost nor cruise time control. Hereafter, Alternative1 refers to the model, which considers fuel and $\mathrm{CO}_{2}$ emission cost but not cruise time control, and Alternative2 refers to the model, which considers neither fuel nor $\mathrm{CO}_{2}$ emissions cost nor cruise time control.

In Table 9, we present the total cost improvements provided by the cruise time control strategy over 23 flight network. When all the results are considered, it is seen that cruise time control can achieve $8 \%$ and $10 \%$ average cost improvement over Alternative1 and Alternative2, respectively. When we examine the effect of different factor levels, the cost improvement of cruise time control is remarkably increasing depending on factor $C$. That means, when higher utilizations of aircraft are more valuable, cruise time control is getting more crucial. The essence of the matter is that cruise time control can decrease the total cost around $9 \%$ and this average improvement can increase up to $11 \%$ when the daily usage cost is higher.

What if the same service levels with the published schedule are used?

Another critical question that might arise is whether the cost improvements over published schedule are achieved by the effect of service level changes in the proposed schedule or not. While comparing the proposed schedule and the published schedule previously, we take the service level as a factor of the experimental design. However, in this part, we use the same minimum service levels with the published schedule on each passenger connection in the proposed schedule.

In Table 10, it is seen that there is around $14 \%$ improvement on the average over the published schedule even though we use the same service levels on passenger connections in the proposed schedule. This improvement is achieved by considering cruise time change along with the fleeting and assignment decisions in the integration of these subproblems. Therefore, it can be deduced that although the same service levels of the published schedule are used, it is possible to improve the total cost by using this novel approach.

\subsection{Analysis on heuristic methods}

In this section, we analyze the proposed heuristic methods in terms of gap and improvement. Initially, the gap between the integrated model and the heuristic methods are examined with 23 flight network. We calculate the gap between the optimal solution of the integrated model and the solution of heuristic methods as follows:

Table 9

Total cost improvement of cruise speed control.

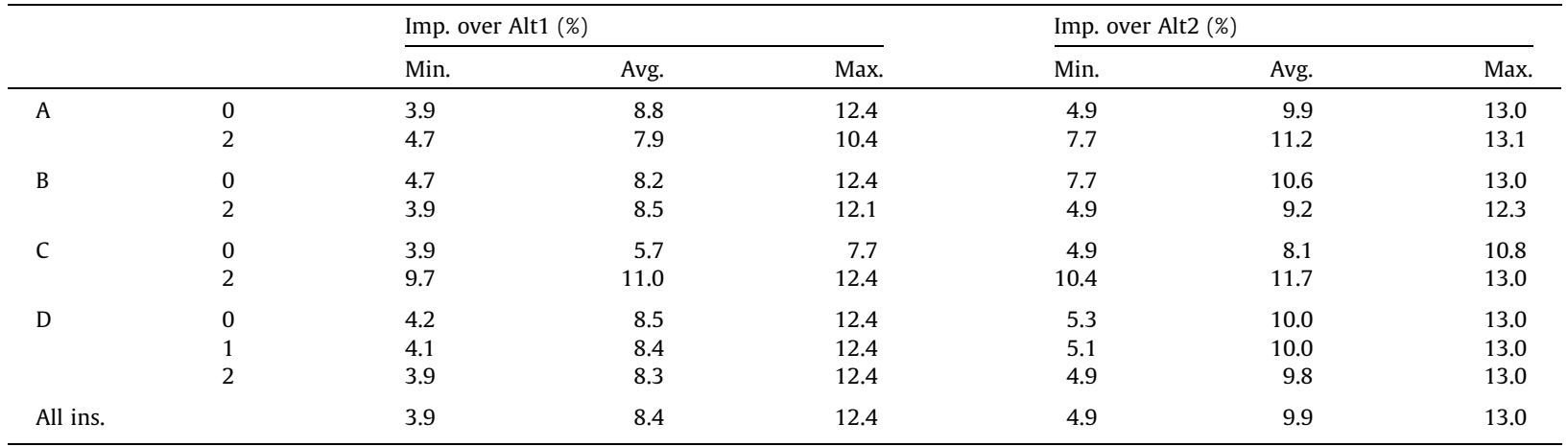

Table 10

Cost improvement over the published schedule with same service levels.

\begin{tabular}{|c|c|c|c|c|c|c|c|c|c|c|c|c|c|}
\hline & & \multicolumn{3}{|c|}{ Fuel and $\mathrm{CO}_{2}(\%)$} & \multicolumn{3}{|c|}{ Idle time (\%) } & \multicolumn{3}{|c|}{ Daily usage (\%) } & \multicolumn{3}{|c|}{ Total (\%) } \\
\hline & & Min. & Avg. & Max. & Min. & Avg. & Max. & Min. & Avg. & Max. & Min. & Avg. & Max. \\
\hline \multirow[t]{2}{*}{ A } & 0 & 4.1 & 5.8 & 14.5 & 52.7 & 60.6 & 62.2 & 0 & 12.3 & 14.7 & 10.2 & 14.2 & 16 \\
\hline & 2 & 4.1 & 9.3 & 14.5 & 52.8 & 57.5 & 62.2 & 0 & 7.4 & 14.7 & 9.7 & 13.3 & 15.4 \\
\hline \multirow[t]{2}{*}{ B } & 0 & 4.1 & 7.6 & 14.5 & 52.8 & 59.1 & 62.2 & 0 & 9.8 & 14.7 & 13.3 & 14.7 & 16 \\
\hline & 2 & 4.1 & 7.5 & 14.5 & 52.7 & 59 & 62.2 & 0 & 9.8 & 14.7 & 9.7 & 12.8 & 14.5 \\
\hline \multirow[t]{2}{*}{ C } & 0 & 4.2 & 11 & 14.5 & 52.7 & 55.9 & 62.2 & 0 & 4.9 & 14.7 & 9.7 & 13.8 & 16 \\
\hline & 2 & 4.1 & 4.2 & 4.2 & 62.2 & 62.2 & 62.2 & 14.7 & 14.7 & 14.7 & 12.1 & 13.7 & 15 \\
\hline \multicolumn{2}{|c|}{ All ins. } & 4.1 & 7.6 & 14.5 & 52.7 & 59 & 62.2 & 0 & 9.8 & 14.7 & 9.7 & 13.7 & 16 \\
\hline
\end{tabular}


Table 11

Gap of heuristic methods over 23 flight network.

\begin{tabular}{|c|c|c|c|c|c|c|c|}
\hline & & \multicolumn{3}{|c|}{ Heuristic 1 (\%) } & \multicolumn{3}{|c|}{ Heuristic 2 (\%) } \\
\hline & & Min. & Avg. & Max. & Min. & Avg. & Max. \\
\hline \multirow[t]{2}{*}{ A } & 0 & 0.000 & 0.000 & 0.001 & 0.000 & 0.3 & 2.1 \\
\hline & 2 & 0.000 & 0.000 & 0.002 & 0.000 & 0.4 & 3.7 \\
\hline \multirow[t]{2}{*}{ B } & 0 & 0.000 & 0.000 & 0.002 & 0.000 & 0.5 & 3.7 \\
\hline & 2 & 0.000 & 0.000 & 0.002 & 0.000 & 0.2 & 2.1 \\
\hline \multirow[t]{2}{*}{ C } & 0 & 0.000 & 0.000 & 0.002 & 0.000 & 0.7 & 3.7 \\
\hline & 2 & 0.000 & 0.000 & 0.000 & 0.000 & 0.000 & 0.001 \\
\hline \multirow[t]{3}{*}{ D } & 0 & 0.000 & 0.000 & 0.001 & 0.000 & 0.4 & 3.7 \\
\hline & 1 & 0.000 & 0.000 & 0.000 & 0.000 & 0.3 & 3.7 \\
\hline & 2 & 0.000 & 0.000 & 0.002 & 0.000 & 0.2 & 2.1 \\
\hline \multicolumn{2}{|c|}{ All ins. } & 0.000 & 0.000 & 0.002 & 0.000 & 0.3 & 3.7 \\
\hline
\end{tabular}

Table 12

Improvement of heuristic methods over 35 flight network.

\begin{tabular}{|c|c|c|c|c|c|c|c|}
\hline & & \multicolumn{3}{|c|}{ Heuristic 1 (\%) } & \multicolumn{3}{|c|}{ Heuristic 2 (\%) } \\
\hline & & Min. & Avg. & Max. & Min. & Avg. & Max. \\
\hline \multirow[t]{2}{*}{ A } & 0 & 0.000 & 3.4 & 14.9 & -0.041 & 3.3 & 14.9 \\
\hline & 2 & 0.000 & 2.3 & 14.0 & -0.291 & 2.1 & 13.6 \\
\hline \multirow[t]{2}{*}{ B } & 0 & 0.000 & 2.6 & 14.9 & -0.149 & 2.4 & 14.9 \\
\hline & 2 & 0.000 & 3.0 & 13.9 & -0.291 & 3 & 13.6 \\
\hline \multirow[t]{2}{*}{ C } & 0 & 0.000 & 2.8 & 13.9 & -0.291 & 2.5 & 13.6 \\
\hline & 2 & 0.000 & 2.9 & 14.9 & -0.149 & 2.9 & 14.9 \\
\hline \multirow[t]{3}{*}{ D } & 0 & 0.000 & 3.1 & 14.9 & -0.291 & 2.9 & 14.9 \\
\hline & 1 & 0.000 & 3.1 & 13.9 & -0.128 & 2.9 & 13.6 \\
\hline & 2 & 0.000 & 2.4 & 13.2 & -0.149 & 2.2 & 13.2 \\
\hline All ins. & & 0.000 & 2.9 & 14.9 & -0.291 & 2.8 & 14.9 \\
\hline
\end{tabular}

Table 13

Gap between the heuristic methods and best lower bound over 35 flight network.

\begin{tabular}{|c|c|c|c|c|c|c|c|}
\hline & & \multicolumn{3}{|c|}{ Heuristic 1 (\%) } & \multicolumn{3}{|c|}{ Heuristic 2 (\%) } \\
\hline & & Min. & Avg. & Max. & Min. & Avg. & Max. \\
\hline A & $\begin{array}{l}0 \\
2\end{array}$ & $\begin{array}{l}-5.783 \\
-6.203\end{array}$ & $\begin{array}{l}-2.148 \\
-2.655\end{array}$ & $\begin{array}{l}0.000 \\
0.000\end{array}$ & $\begin{array}{l}-6.002 \\
-6.690\end{array}$ & $\begin{array}{l}-2.218 \\
-2.859\end{array}$ & $\begin{array}{l}0.000 \\
0.000\end{array}$ \\
\hline B & $\begin{array}{l}0 \\
2\end{array}$ & $\begin{array}{l}-6.203 \\
-5.707\end{array}$ & $\begin{array}{l}-2.601 \\
-2.202\end{array}$ & $\begin{array}{r}0.000 \\
-0.002\end{array}$ & $\begin{array}{l}-6.690 \\
-5.722\end{array}$ & $\begin{array}{l}-2.828 \\
-2.248\end{array}$ & $\begin{array}{r}0.000 \\
-0.003\end{array}$ \\
\hline C & $\begin{array}{l}0 \\
2\end{array}$ & $\begin{array}{l}-6.203 \\
-1.231\end{array}$ & $\begin{array}{l}-4.200 \\
-0.603\end{array}$ & $\begin{array}{r}0.000 \\
-0.002\end{array}$ & $\begin{array}{l}-6.690 \\
-1.406\end{array}$ & $\begin{array}{l}-4.429 \\
-0.647\end{array}$ & $\begin{array}{r}0.000 \\
-0.003\end{array}$ \\
\hline $\mathrm{D}$ & $\begin{array}{l}0 \\
1 \\
2\end{array}$ & $\begin{array}{l}-5.094 \\
-5.059 \\
-6.203\end{array}$ & $\begin{array}{l}-2.091 \\
-2.279 \\
-2.835\end{array}$ & $\begin{array}{r}0.000 \\
0.000 \\
-0.188\end{array}$ & $\begin{array}{l}-5.650 \\
-5.346 \\
-6.690\end{array}$ & $\begin{array}{l}-2.244 \\
-2.407 \\
-2.964\end{array}$ & $\begin{array}{r}0.000 \\
0.000 \\
-0.188\end{array}$ \\
\hline \multicolumn{2}{|c|}{ All ins. } & -6.203 & -2.470 & -0.002 & -6.690 & -2.611 & -0.003 \\
\hline
\end{tabular}

$$
\text { Gap }=\frac{\text { Heuristic }- \text { Optimal }}{\text { Optimal }}
$$

As it can be seen from Table 11, the gaps of the heuristic methods are less than $1 \%$ on the average at all instances. It is worthwhile to point out that Heuristic 1 has $0.002 \%$ maximum gap over all factor levels and all instances. Although, Heuristic 2 has maximum gap 3.7\% over all instances, when factors C and B are at their high levels, it has the maximum gap of $0.001 \%$ and $2.1 \%$, respectively. This is due to the fact that Heuristic 2 fixes the routings and fleeting decisions by just considering daily usage and spill cost. When these cost terms dominate the other cost terms on routings and fleeting decisions, ignoring fuel and $\mathrm{CO}_{2}$ emission and idle time costs at routing and fleeting gets less significant. Therefore, Heuristic 2 causes smaller gaps when factors $\mathrm{B}$ and $\mathrm{C}$ are at their high levels and factor $\mathrm{A}$ is at the low level. 
Afterwards, we compare the performances of heuristic methods with the integrated model over 35 flight network to see a possible impact of a larger flight network. Due to computational requirements of the integrated model, we put a time limit of $5400 \mathrm{~s}$ for the exact approach and report the best incumbent solution generated by the integrated model in a given time limit. Consequently, we calculate the improvement of solutions of heuristic methods over the best solution as follows:

$$
\text { Improvement }=\frac{\text { Cost of the Best Incumbent Solution }- \text { Cost of the Heuristic Method }}{\text { Cost of the Heuristic Method }}
$$

As reported in Table 12, both heuristic methods now surpass the best incumbent solution of the integrated model on the average due to the time limit of $5400 \mathrm{~s}$. While Heuristic 1 achieves $2.9 \%$, Heuristic 2 achieves $2.8 \%$ average improvement over all instances. The negative improvement in Heuristic 2 means that the best solution generated in $5400 \mathrm{~s}$ by the integrated model has smaller objective value than Heuristic 2 for certain instances.

In Table 13, we compare the cost of the heuristic methods and the cost of the best upper bound obtained in $5400 \mathrm{~s}$. However, the optimal value of the integrated model is between the best upper bound and the best lower bound. Therefore, we also provide a comparison of the performance of the proposed heuristic methods against the best lower bound obtained by the commercial solver in $5400 \mathrm{~s}$. The gap between the cost of the heuristic methods and the best lower bound value is found by the following equation.

$$
\text { Gap }=\frac{\text { Best Lower Bound }- \text { Cost of the Heuristic Method }}{\text { Cost of the Heuristic Method }}
$$

It is worthwhile to note that the gap between the heuristic methods and the best lower bound is approximately $3 \%$ on the average for both heuristic methods. Together with the analysis over the best incumbent solution, this observation implies that the heuristic methods perform quite close to the integrated model over the 35 flight network as well as for the 23 flight network.

Finally, we analyze the heuristic methods over the 114 flight network. As discussed earlier, the integrated model cannot find a feasible solution in six hours so we compare the performance of the heuristic methods over the cost of published schedule in Table 14. It can be seen that both heuristic methods provide around $15 \%$ average improvement over all instances in comparison to the published schedule.

In the proposed model, for constraint (3.26), we take the minimum desired service levels for passenger connections, $\gamma_{i j}$, equal to the levels of factor D, e.g. $75 \%, 85 \%$ and $95 \%$, respectively. On the other hand, we could also calculate the probability of successful passengers' connection for each connecting passenger, denoted as $\beta_{i j}$, by replacing Constraint (3.7) with the following constraints:

$$
\begin{aligned}
& \operatorname{Pr}\left[N C_{i} \leqslant d_{j}-d_{i}-\sum_{t \in T} f_{i}^{t}-T P_{i j}\right] \leqslant \beta_{i j} \quad \forall i \in F, j \in P_{i} \\
& \beta_{i j} \geqslant \gamma_{i j} \quad \forall i \in F, j \in P_{i}
\end{aligned}
$$

\begin{tabular}{|c|c|c|c|c|c|c|c|}
\hline & & \multicolumn{3}{|c|}{ Heuristic 1 (\%) } & \multicolumn{3}{|c|}{ Heuristic $2(\%)$} \\
\hline & & Min. & Avg. & Max. & Min. & Avg. & Max \\
\hline \multirow[t]{2}{*}{ A } & 0 & 10.6 & 17.9 & 25.9 & 10.6 & 16.7 & 25.9 \\
\hline & 2 & 9.8 & 14.6 & 19.8 & 9.2 & 12.3 & 15.9 \\
\hline \multirow[t]{2}{*}{ B } & 0 & 11.3 & 17.1 & 25.9 & 9.8 & 14.9 & 23.5 \\
\hline & 2 & 9.8 & 15.4 & 24.5 & 9.2 & 14.1 & 22.7 \\
\hline \multirow[t]{2}{*}{$\mathrm{C}$} & 0 & 14.6 & 21.0 & 25.9 & 12.2 & 18.3 & 23.5 \\
\hline & 2 & 9.8 & 11.5 & 12.4 & 9.2 & 10.7 & 11.9 \\
\hline \multirow[t]{3}{*}{$\mathrm{D}$} & 0 & 9.9 & 16.4 & 25.9 & 9.4 & 14.6 & 23.5 \\
\hline & 1 & 9.8 & 16.3 & 25.9 & 9.3 & 14.6 & 23.4 \\
\hline & 2 & 9.8 & 16.0 & 25.3 & 9.2 & 14.2 & 22.6 \\
\hline All ins. & & 9.8 & 16.2 & 25.9 & 9.2 & 14.5 & 23.5 \\
\hline
\end{tabular}

Table 14

Improvement of heuristic methods over the published schedule in 114 flight network.

\begin{tabular}{|c|c|c|c|c|c|c|c|c|c|}
\hline & \multicolumn{3}{|c|}{ Integrated model (in sec) } & \multicolumn{3}{|c|}{ Heuristic 1 (in sec) } & \multicolumn{3}{|c|}{ Heuristic 2 (in sec) } \\
\hline & Min. & Avg. & Max. & Min. & Avg. & Max. & Min. & Avg. & Max. \\
\hline $23 \mathrm{FN}$ & 18 & 968 & 4048 & 0.8 & 1.1 & 1.8 & 10.5 & 12.4 & 15.3 \\
\hline $35 \mathrm{FN}$ & 4243.4 & 5381.1 & 5400 & 3.2 & 5.7 & 7.8 & 31.6 & 40.2 & 54.7 \\
\hline $114 \mathrm{FN}$ & - & - & - & 724.8 & 5173.4 & 5400 & 1702.6 & 3254.3 & 5400 \\
\hline
\end{tabular}

Table 15

CPU time analysis. 
Consequently, the achieved service levels of the proposed schedule are around $98 \%, 98 \%$ and $99 \%$, respectively, on the average. However in the published schedule, each connection has a different service level and some of them are actually lower than even $75 \%$, which is the lowest level of factor D in the computational study.

In summary, both heuristic methods have quite small optimality gaps over 23 flight network; while heuristic methods surpass the best solution of the integrated model in 5400 s over 35 flight networks. Moreover, heuristic methods work quite faster than the integrated model in terms of the CPU time. When the performance of the heuristic methods are compared, it can be said that Heuristic 1 surpasses the Heuristic 2 in terms of optimality gap and improvement over the best solution and published schedule over all flight networks. In Table 15, even for the CPU time, Heuristic 1 is more favorable than Heuristic 2 in 23 and 35 flight networks, however in 114 flight the CPU time of heuristic2 is 2000 s less than the CPU time of Heuristic 1 on the average, which could be attributed to the higher number of binary variables due to the discretization step in Heuristic 1. In other words, in 114 flight network even if the improvement of Heuristic 2 is $1.8 \%$ smaller than the improvement of Heuristic 1, Heuristic 2 works 2000 s faster on the average.

\section{Conclusion}

For an integration of robust airline scheduling, aircraft fleeting and routing problems, this novel consideration of cruise speed/time control enables us to make following contributions. First, aircraft utilization could be evaluated in terms of considering the entire flight network. We also show that total number of aircraft needed to cover a set of flights could be decreased while ensuring equivalent service level and maintenance requirements. Therefore, there is a significant tradeoff between aircraft utilization and overall operational expenses. Moreover, due to a possible increase in the utilization of a fuel efficient aircraft, total cost of fuel consumption could be decreased for the entire flight network. Second, the robustness can be achieved with smaller cost. To elaborate, on a route having a flight with a great delay probability would require idle time insertion for the following flights to be performed on time; while removing the problematic flight from that sequence could render that intervention unnecessary. Due to cruise speed control, our study has more options to make this type of changes on routing decisions.

In addition to the contributions due to cruise speed control, we put forward the following contributions regarding the solution of the problem. First, we propose a nonlinear mixed integer programming model and present its second order conic reformulation. Furthermore, to solve large scale problems in a reasonable computation time, we propose two heuristic methods, which are discrete approximation and cruise speed control algorithm, and multi-stage triplet search algorithm. Finally, we conduct a computational study in which we analyze these three proposed solution methods and compare their performances among each other and against the published schedule.

Since we deal with the integration of three airline schedule planning problems, one immediate future research direction can be integration of the last problem, crew assignment problem. Although there are different studies proposed to integrate the crew assignment problem to one of these three stages; as far as we know, there is not a study which integrates these four problems within the consideration of passengers' service levels and cruise speed control.

\section{Acknowledgments}

The authors thank the editor and three anonymous referees for their constructive comments and suggestions that significantly improved this paper.

\section{References}

Aktürk, M.S., Atamtürk, A., Gürel, S., 2009. A strong conic quadratic reformulation for machine-job assignment with controllable processing times. Oper. Res. Lett. 37 (3), 187-191.

Aktürk, M.S., Atamtürk, A., Gürel, S., 2014. Aircraft rescheduling with cruise speed control. Oper. Res. 62 (4), $829-845$.

Aloulou, M.A., Haouari, M., Mansour, F.Z., 2013. A model for enhancing robustness of aircraft and passenger connections. Transport. Res. Part C: Emerg. Technol. 32, 48-60.

Arikan, M., Deshpande, V., Sohoni, M., 2013. Building reliable air-travel infrastructure using empirical data and stochastic models of airline networks. Oper. Res. 61 (1), 45-64.

Barnhart, C., Cohn, A., 2004. Airline schedule planning: accomplishments and opportunities. Manuf. Serv. Oper. Manage. 6 (1), $3-22$.

Barnhart, C., Belobaba, P., Odoni, A.R., 2003. Applications of operations research in the air transport industry. Transport. Sci. 37 (4), $368-391$.

Boeing, Jet Prices, 2015. <http://www.boeing.com/boeing/commercial/prices/> (Visited July 2015).

BTS, 2010. Airline On-Time Performance Data. <http://www.transtats.bts.gov/DL_SelectFields.asp?Table_ID=236\&DB_Short_Name=On-Time> (Visited June 2015).

Duran, A.S., Gürel, S., Aktürk, M.S., 2015. Robust airline scheduling with controllable cruise times and chance constraints. IIE Trans. 47 (1), 64-83.

EUROCONTROL, 2012. User Manual for the Base of Aircraft Data (bada) Revision 3.10, Technical Report 12/04/10-45, EUROCONTROL Experimental Centre Centre de Bois des Bordes B.P.15 F - 91222 Brtigny-sur-Orge CEDEX FRANCE.

Günlük, O., Linderoth, J., 2010. Perspective reformulations of mixed integer nonlinear programs with indicator variables. Math. Program. 124 (1-2), 183205.

Haouari, M., Shao, S., Sherali, H.D., 2013. A lifted compact formulation for the daily aircraft maintenance routing problem. Transport. Sci. 47 (4), 508-525. Hiriart-Urruty, J.-B., Lemaréchal, C., 2001. Fundamentals of Convex Analysis. Springer.

IATA, 2010. Airline Fuel and Labor Cost Share. <http://www.iata.org/whatwedo/Documents/economics/Airline_Labour_Cost_Share_Feb2010.pdf> (Visited June 2015)

Lohatepanont, M., Barnhart, C., 2004. Airline schedule planning: integrated models and algorithms for schedule design and fleet assignment. Transport. Sci. 38 (1), 19-32. 
Parmentier, A., 2013. Aircraft Routing: Complexity and Algorithms, Master's thesis, Ecole des Ponts ParisTech.

Rebollo, J.J., Balakrishnan, H., 2014. Characterization and prediction of air traffic delays. Transport. Res. Part C: Emerg. Technol. 44, 231-241.

Ryerson, M.S., Hansen, M., 2013. Capturing the impact of fuel price on jet aircraft operating costs with leontieff technology and econometric models, Transport. Res. Part C: Emerg. Technol. 33, 282-296.

Sherali, H.D., Bae, K.-H., Haouari, M., 2013a. A benders decomposition approach for an integrated airline schedule design and fleet assignment problem with flight retiming, schedule balance, and demand recapture. Ann. Oper. Res. 210 (1), 213-244.

Sherali, H.D., Bae, K.-H., Haouari, M., 2013b. An integrated approach for airline flight selection and timing, fleet assignment, and aircraft routing. Transport. Sci. 47 (4), 455-476. 